Article

\title{
Identifying Significant Risks and Analyzing Risk Relationship for Construction PPP Projects in China Using Integrated FISM-MICMAC Approach
}

\author{
Xiaoyan Jiang ${ }^{1, * \mathbb{C}}, \mathrm{Kun} \mathrm{Lu}^{1}{ }^{1}$, Bo $\mathrm{Xia}^{2}{ }^{2}$, Yong $\mathrm{Liu}^{3}$ and Caiyun Cui ${ }^{4}$ \\ 1 School of Civil Engineering, Hefei University of Technology, Hefei 230009, Anhui, China; \\ lukun@mail.hfut.edu.cn \\ 2 School of Civil Engineering and Built Environment, Queensland University of Technology, Gardens Point, \\ Brisbane, QLD4001, Australia; paul.xia@qut.edu.au \\ 3 School of Civil Engineering and Architecture, Zhejiang Sci-Tech University, Hangzhou 310018, China; \\ jhly1007@163.com \\ 4 North China Institute of Science and Technology, Langfang 065201, China; cuicaiyun@163.com \\ * Correspondence: jiangxiaoyan@hfut.edu.cn
}

Received: 30 August 2019; Accepted: 17 September 2019; Published: 23 September 2019

\begin{abstract}
To meet the growing demand for public facilities and services, many developing countries, including China, have adopted the concept of public-private partnership (PPP). However, there are many risks in PPP projects. Furthermore, these risks affect each other, which may lead to project failure. However, the existing research on the PPP risk relationship has not gone into sufficient detail. Therefore, in order to fill this literature gap, this study proposes a procedural method to analyze the correlation between PPP risks. Firstly, this study, identifies the risks of construction PPP projects in China by combining the literature review with a case study and interviews. Then, fuzzy interpretative structural modeling (FISM) is used to reflect the relationships between these risks and reveal the failure mechanisms of PPP projects. In addition, based on matrix impact cross-reference multiplication applied to a classification (MICMAC) analysis, the risk is divided into four clusters, according to the driving and dependence power, to show the relationship level of the risk. Finally, the paper compares and discusses the research results with other studies and puts forward some suggestions on PPP risks. The FISM-MICMAC method adopted in this study considers the fuzzy of the PPP risk relationship and improves upon previous studies. In addition, the method of FISM-MICMAC can provide a new risk assessment tool for risk management strategies in the field of construction engineering and management.
\end{abstract}

Keywords: Public-private partnership (PPP); risk identification; risk relationship; triangular fuzzy number; ISM; MICMAC

\section{Introduction}

A PPP (public-private partnership) refers to a partnership between the government and private investors to provide public infrastructure projects, public goods, and services [1,2]. PPP originated in the UK [3]. As it can deliver high-quality results within the concession period and budget [4], PPP has attracted extensive attention from the public sector and has been adopted in a number of countries [5-7].

However, PPP projects are characterized by large scale investments, long contract concession periods, and complex technologies, which give rise to many potential risk factors in the implementation process, which could also lead to the failure of PPP projects [8-10]. According to the World Bank, 279 PPP projects have been "Cancelled" since 1990 [5]. 
In the wake of the 2007-2008 global financial crisis, the Chinese government has become increasingly interested in PPP as a way for local governments to transfer their debts [10]. However, compared with developed countries, the growth of PPP in China is still in its infancy [11]. Furthermore, some PPP projects cannot be successful in China [12,13]. As a result, on 16 November 2017, China's Ministry of Finance announced the "Notice of standardizing project library in PPP integrated information platform" (hereafter referred to as "No. 92") [14], designed to correct the current problem of deviation and variation in the course of PPP project implementation, further improve the quality of the project library storage, as well as project effectiveness and information given to the public, and improved social supervision. Since the release of No. 92, from 16 November 2017 to 16 August 2019, the number of "withdrawal projects" reduced by the PPP project library of the Ministry of Finance has reached 6955, and there are 12,561 existing PPP projects in the PPP project library [15].

PPP project failures and cost overruns are not rare, and they pose a threat to sustainable development [16]. The failure of a PPP is largely due to PPP risks [10,17]. There are many risks in PPP projects which, furthermore, are correlated $[18,19]$. According to accident causal chain theory, accidents are usually caused by a chain of events in the system and the sequential occurrence of causality $[20,21]$. The failure of PPP projects is usually not caused by a single risk factor, but by a single risk inducing other risks, forming a chain of PPP project risks and, finally, leading to the failure of the PPP [18,19].

Existing studies have mainly focused on the key success factors of PPP [10], risk factors [18,22], risk allocation [17,23], and risk assessment [11,24], etc. However, no in-depth studies on the relationships between PPP risks have been carried out. It is crucial to know what relationships exist between PPP risks, and which risks play a leading role in triggering project failure, in order to help PPP managers to better understand the impact of the relationship between risks on project failure, and to distinguish the key points in future risk management and control.

This study identifies the key risk factors of PPP projects by literature survey, case study, and interviews. Then, fuzzy interpretative structural modeling (FISM), which combines fuzzy theory and interpretative structural modeling (ISM), is used to reflect the relationships between these risks and reveal the failure mechanisms of PPP projects. In addition, key risk factors are classified with the MICMAC (matrix impact cross-reference multiplication applied to a classification) method, and the driving effect of each risk factor on the failure of PPP project is analyzed. Finally, the similarities and differences between this study and other studies are analyzed, and suggestions for managing key risk factors of PPP projects are given.

\section{Literature Review}

\subsection{General Review of PPP Risks}

The impact of risks on the completion of a PPP project is usually significant [11]. In PPP projects, various risks exist, not only due to the complexity of the financial and organizational structures of the project, but also due to the large amount of investment, long operation period, sophisticated technical know-how of the project, political impact, and government involvement [25].

The existing studies on PPP risks have mainly been focused on risk factors, risk allocation, risk assessment, and risk identification, etc. Only a limited number of studies have investigated the relationships of PPP risks. Some researchers have sought to identify the risk factors associated with PPPs in specific projects or in specific countries [26,27], and have generally categorized them in terms of being equally shared by both parties or mostly allocated either to the public or private partners [28]. In addition, many scholars have also studied proper allocation of risk factors between the project participants $[1,29,30]$. Jin combined fuzzy logic with artificial neural network techniques to design a neuro-fuzzy model to help the effective allocation of risks in PPP projects [31]. Ameyaw and Chan's approach based on fuzzy set theory outlined the risk allocation principle, explained the fuzziness inherent in the human cognitive process, and made a case study of risk allocation in a PPP water supply project in Ghana [32]. According to the findings of Jayasuriya et al., there has often been disagreement 
between PPP regulators (public partner) and operators (private partner) about the preferred risk allocation [28]. For PPP risk assessment, efforts have also been made to develop models to evaluate PPP risk values $[11,24,33,34]$. Li and Zou applied a fuzzy analytic hierarchy process (FAHP) to the risk assessment of PPP projects [24]. Mazher et al. proposed a PPP infrastructure project risk assessment method based on a fuzzy measure and a non-additive fuzzy integral [35]. Thomas et al. proposed a risk probability and impact assessment framework for build-operate-transfer (BOT) roads, based on a fuzzy-fault tree and the Delphi method [36]. Bai et al., based on the methods of fuzzy comprehensive evaluation model and failure mode, conducted an effects and criticality analysis for evaluating the sustainability risk level of PPP projects [37].

PPP risks identification and relationships among PPP risks will be described in the following subsections.

\subsection{Identification Methods of PPP Risks}

As mentioned, some scholars have systematically reviewed the risk factors. PPP risks are specific for different types of projects. For sponge city projects [38], water projects [29], highway projects [39], waste-to-energy projects [26], marine projects [40], and other project types, the inherent PPP risks are different. In addition, for different countries, such as Indonesia [41], China [26], the United States [42], Iran [9], and Malaysia [43], the PPP risks are also different.

Due to the specificity of PPP project risks, specific risks should be extracted for different types of PPP projects in different countries. Some scholars and risk managers have attempted to introduce a variety of different risk identification methods into PPP projects, among which the three most frequently used risk identification methods are listed in Table 1.

Table 1. Common methods for identifying public-private partnership (PPP) risks.

\begin{tabular}{|c|c|c|c|}
\hline Method & Advantage & Disadvantage & Reference \\
\hline Interview method & $\begin{array}{l}\text { Experts have rich experience } \\
\text { and knowledge, which is } \\
\text { conducive to the rapid } \\
\text { identification of risks. }\end{array}$ & $\begin{array}{l}\text { Subject to the subjective } \\
\text { influence of experts; experts } \\
\text { require higher ability. }\end{array}$ & $\begin{array}{l}\text { Xu et al. [30] } \\
\text { Ke et al. [17] } \\
\text { Chan et al. [44] } \\
\text { Ke et al. [45] }\end{array}$ \\
\hline Literature survey & $\begin{array}{l}\text { Risk identification is easy to } \\
\text { operate and is low cost, } \\
\text { which is conducive to } \\
\text { reducing the interference of } \\
\text { subjective factors. }\end{array}$ & $\begin{array}{l}\text { It is necessary to consider } \\
\text { the influence of different } \\
\text { industrial categories, } \\
\text { regions, and other factors on } \\
\text { risk identification. }\end{array}$ & $\begin{array}{l}\text { Osei-Kyei et al. [10] } \\
\text { Xu et al. [11] } \\
\text { Ameyaw et al. [46] } \\
\text { Ameyaw et al. [47] }\end{array}$ \\
\hline Case study & $\begin{array}{l}\text { High credibility with the } \\
\text { actual situation. }\end{array}$ & $\begin{array}{l}\text { Case collection and analysis } \\
\text { requires much time } \\
\text { and money. }\end{array}$ & $\begin{array}{c}\text { Xu et al. [48] } \\
\text { Ameyaw et al. [46] } \\
\text { Ameyaw et al. [47] }\end{array}$ \\
\hline
\end{tabular}

\subsection{Relationship among PPP Risks}

From the above analysis, it is evident that there are relationships between PPP risks that lead to the mutual influence of risks $[18,19]$. In analyzing risks at different project stages, it is important to consider their interrelationships, because an understanding of the relationships between the risks can facilitate a more holistic risk identification and assessment. The existence of risk at an earlier stage may contribute to increased risk manifesting in later stages, or it may not. Sometimes a less important risk can affect a more significant risk, both in terms of likelihood and severity [49].

For example, Valipour, A. et al. identify PPP shared risks using an approach in the form of a hybrid Fuzzy method and Cybernetic Analytic Network Process (CANP) model [50]. Wang et al. proposed a risk model, named the Alien Eye's Risk Model, to show the hierarchical levels of the risks and the influential relationship among the risks in a risk influence matrix [51]. Aladağ and Işık used FAHP to determine the priority factors of design and construction risks in build-operate-transfer (BOT) type mega transportation projects [52]. Furthermore, a fuzzy analytic network process (FANP) 
method was applied for overcoming the problems of interdependencies and feedback among different risk-ranking alternatives in freeway PPP projects [53]. Multiple-regression analysis was also proposed to estimate and quantify risk interrelationship [54].

\subsection{Research Gap and Contribution}

These studies have used techniques, such as fuzzy synthetic evaluation [11], fuzzy analytic hierarchy processes [24,33], 2-dimensional linguistic information [34], ISM-MICMAC method [19], hybrid fuzzy cybernetic analytic network process model [50] and multiple-regression analysis [54] etc., to analyze PPP risks and their relationships. However, studies on these relationships and influences based on failed PPP cases in China is lacking. Moreover, it is common to utilize a single approach, but less common to utilize a fuzzy-ISM integrated MICMAC method comprehensively in PPP risks relationship analysis.

The main contribution of the study is to present the application of the fuzzy-ISM technique integrated MICMAC analysis on the relationship among significant PPP risks, which affect the implementation of PPP projects in China, and also to analyze which risks play a leading role in triggering project failure. The 28 cases in this study also make an important contribution to PPP case studies, because failed PPP projects in China can be used as important references for other countries. In addition, this study makes a contribution to relevant literature on common methods for identifying PPP risks and to our understanding of failed PPP projects by summarizing some abnormal phenomena, such as government buyback, project predicament or no operations, severe losses, management right transfers, failure to realize value for money (VFM), and contract cancellations.

\section{Research Methods}

This study draws on literature, case studies and expert interviews to collect data, and identifies the significant PPP risks in China. Then, the fuzzy ISM (FISM) approach is adopted to clarify the interaction relationships among the PPP risks and to establish a hierarchical structure of these risks. Finally, the MICMAC integrated with fuzzy-ISM approach is employed to determine the significant risks from the perspective of their interaction status. There are six analysis steps in this study, as shown in Figure 1. The details of these six steps will be described in the following subsections. 


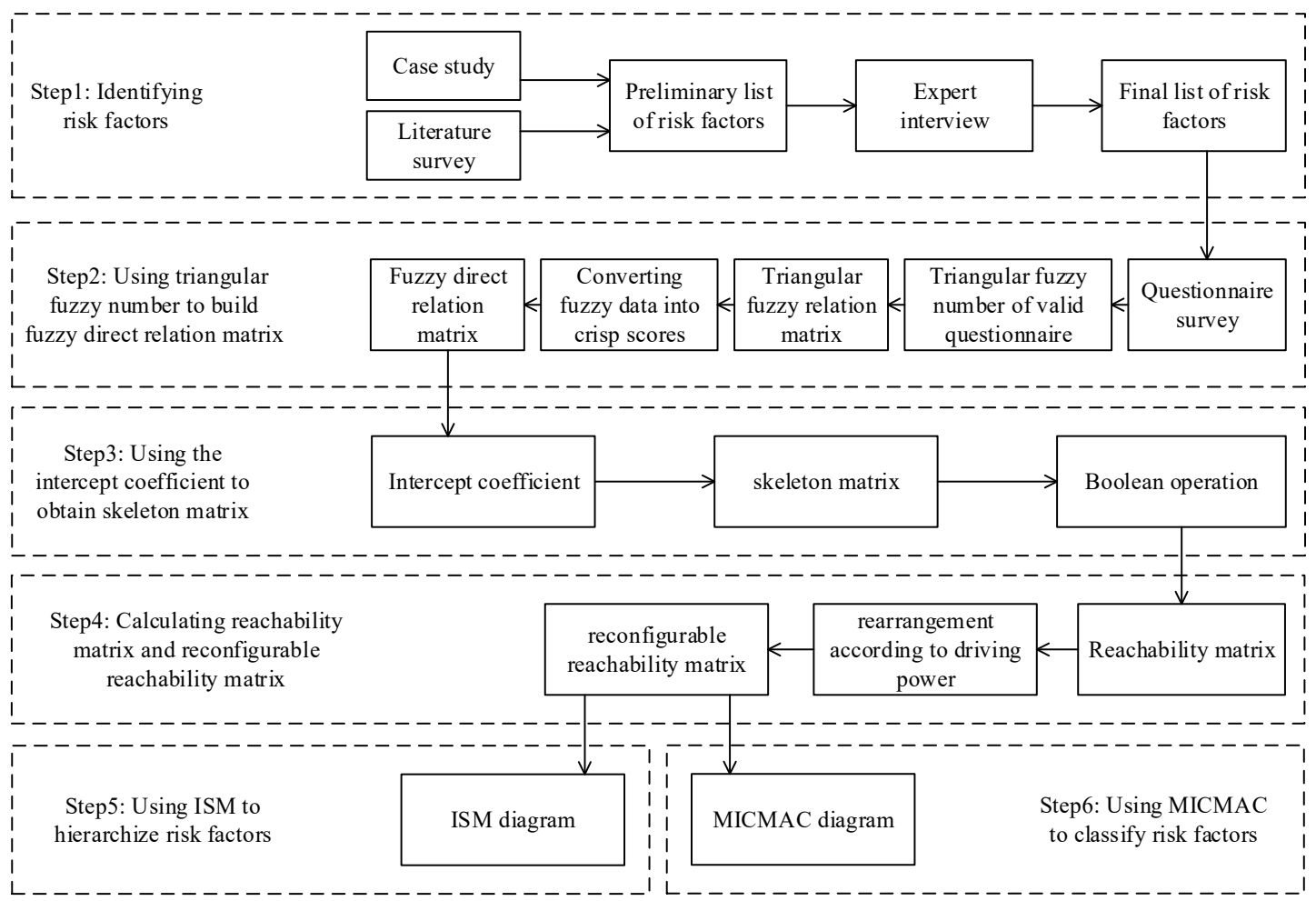

Figure 1. Research framework of the study.

\subsection{Step 1: Collecting Data and Identifying Risk Factors}

The preliminary factor list is extracted by case analysis and literature review, following which, the factor list is modified and simplified by expert interview.

The 28 failed PPP projects are from the World Bank website [55] and China Public-Private Partnerships Center [56]. In order to comply with Chinese national conditions, all of these cases and the literature were based on the actual situation of China. But we cannot find a unified definition on "failed project". Based on prior studies $[57,58]$, this study deems a PPP "failure" as when the following phenomena occur: government buyback, project predicament or no operations, severe losses, management right transfers, failure to realize VFM, and contract cancellations. The cases were chosen as typical examples of the failed PPP projects, with their unique characteristics and failure causes were an excellent match to the critical PPP risk identification. Details of the 28 cases can be found in Appendix A. We have adopted a broadly similar format where possible, in each case drawing out the key risk factors relevant to failure or problem description.

\subsection{Step 2: Using Triangular Fuzzy Number to Build Fuzzy Direct Relation Matrix}

This study utilizes triangular fuzzy number to build fuzzy direct relation matrix. It has been found that fuzzy research, as applied in the construction management discipline, can be divided into two broad fields-fuzzy sets and hybrid fuzzy techniques [59]. The triangular fuzzy number method belongs to the former category [60].

Since the publication of the seminal work "Fuzzy sets" by Zadeh [61], defuzzification by using the fuzzy approach has led to many successful practical applications. For example, Paek applied a fuzzy set approach to price to analyze construction risk [62]. Zhang and Zou used a fuzzy analytic hierarchy process (FAHP) for the appraisal of the risk environment pertaining to joint ventures, in order to support the rational decision-making of project stakeholders [63]. Abdelgawad and Fayek used Fuzzy fault-tree analysis to quantitatively assess risk events in the construction industry [64].

Zadeh proposed the concept of fuzzy sets in order to solve problems described as semi-structured or ill-structured, which allows us to process and transform imprecise information effectively and 
flexibly [57]. Because of the uncertainty of objective things and the fuzziness of human thought, fuzzy decision-making based on fuzzy sets theory has become a basic method for decision-making, meanwhile triangular fuzzy numbers have been extensively applied in fuzzy control and fuzzy decision-making [65-67].

In this study, the fuzzy triangle number of the relationship between various factors is obtained by expert scoring. A triangular fuzzy number is usually represented by three letters: $l, m$, and $r$. These three parameters, respectively, represent the minimum possible value, the median value, and the maximum possible value, (i.e., $l \leq m \leq r$ ) [49]. Referring to the value table of language operators and triangular fuzzy numbers given by Li [68] (as Table 2), we let experts judge the strength of the relationship between the two risk factors.

Table 2. Triangular fuzzy number corresponding to language operator.

\begin{tabular}{cc}
\hline Language Operator & Triangular Fuzzy Number \\
\hline Very low impact $(\mathrm{VL})$ & $(0,0,0.25)$ \\
Low impact $(\mathrm{L})$ & $(0,0.25,0.5)$ \\
medium impact $(\mathrm{M})$ & $(0.25,0.5,0.75)$ \\
High impact $(\mathrm{H})$ & $(0.5,0.75,1)$ \\
Very high impact $(\mathrm{VH})$ & $(0.75,1,1)$ \\
\hline
\end{tabular}

According to the results of the questionnaire, a triangular fuzzy relation matrix is established to judge the strength of the relationship between failure risk factors. In triangular fuzzy relation matrix $\widetilde{D}^{k}$, the triangular fuzzy number $\widetilde{d_{i j}^{k}}=\left(l_{i j}^{k}, m_{i j}^{k}, r_{i j}^{k}\right)$ is used to represent the judgment result of the influence degree of the risk factor $R_{i}$ on $R_{j}$, given by the expert (as follows).

$$
\widetilde{D}^{k}=\left[\begin{array}{cccc}
0 & \widetilde{d}_{12}^{k} & \cdots & \widetilde{d}_{1 n}^{k} \\
\widetilde{d_{21}^{k}} & 0 & \cdots & \widetilde{d}_{2 n}^{k} \\
\vdots & \vdots & \vdots & \vdots \\
\widetilde{d_{n 1}^{k}} & \widetilde{d}_{n 2}^{k} & \cdots & 0
\end{array}\right]
$$

Then, the fuzzy direct relation matrix $D$. of failure risk factors can be obtained by converting fuzzy data into crisp scores (CFCS) method [69]. The specific steps are shown below:

1. Standardize the triangular fuzzy number

$$
\begin{gathered}
a_{i j}^{k}=\left(l_{i j}^{k}-\min l_{i j}^{k}\right) / \Delta_{\min }^{\max } \\
b_{i j}^{k}=\left(m_{i j}^{k}-\min m_{i j}^{k}\right) / \Delta_{\min }^{\max } \\
c_{i j}^{k}=\left(r_{i j}^{k}-\min r_{i j}^{k}\right) / \Delta_{\min }^{\max } \\
\Delta_{\min }^{\max }=\max r_{i j}^{k}-\min l_{i j}^{k}
\end{gathered}
$$

2. Calculate the left and right limits of the standardized values $u_{i j}^{k}$ and $v_{i j}^{k}$

$$
\begin{aligned}
& u_{i j}^{k}=b_{i j}^{k} /\left(1+b_{i j}^{k}-a_{i j}^{k}\right) \\
& v_{i j}^{k}=c_{i j}^{k} /\left(1+c_{i j}^{k}-b_{i j}^{k}\right)
\end{aligned}
$$

3. Calculate the total value of the standardized values

$$
w_{i j}^{k}=\frac{u_{i j}^{k}\left(1-u_{i j}^{k}\right)+\left(v_{i j}^{k}\right)^{2}}{1-u_{i j}^{k}+v_{i j}^{k}}
$$

4. Calculate the exact value of expert triangle fuzzy judgment $d_{i j}^{k}$ 


$$
d_{i j}^{k}=\min l_{i j}^{k}+w_{i j}^{k} \cdot \Delta_{\min }^{\max }
$$

5. Calculate the standardized accurate value $d_{i j}$, as evaluated by experts

$$
d_{i j}=\frac{1}{p} \cdot \sum_{k=1}^{p} d_{i j}^{k}
$$

6. Determine the fuzzy direct relation matrix $D$ of key risk factors

$$
D=\left[\begin{array}{cccc}
0 & d_{12} & \cdots & d_{1 n} \\
d_{21} & 0 & \cdots & d_{2 n} \\
\vdots & \vdots & \ddots & \vdots \\
d_{n 1} & d_{n 2} & \cdots & 0
\end{array}\right]
$$

\subsection{Step 3: Using the Intercept Coefficient to Obtain the Skeleton Matrix}

By using appropriate intercept coefficients $\alpha$, the fuzzy direct relation matrix is transformed into a skeleton matrix $S$, which can be directly used for Boolean operation. When the elements in the fuzzy direct relation matrix are greater than or equal to $\alpha$, the corresponding position of the elements in the skeleton matrix is set to 1 ; otherwise it is set to 0 .

$$
s_{i j}=\left\{\begin{array}{l}
1\left(d_{i j} \geq \alpha\right) \\
0\left(d_{i j}<\alpha\right)
\end{array}\right.
$$

\subsection{Step 4: Calculating Reachability Matrix and Reconfigurable Reachability Matrix}

ISM is an effective model that can clearly define inter-relationships between multiple elements of a problem [70]. In order to study the correlation between evaluation objects, Mandal and Deshmukh introduced interpretative structural modeling (ISM) [71]. ISM has become an analytical tool widely used by scholars to study the interdependence and interaction of factors, and is widely used in policy analysis, supply chains and in other fields [72,73]. For example, Tseng et al. used ISM to construct a systematic model of interconnected natural disaster risks [74]. Yanmei et al. identified power overload risk factors and established ISM to analyze the relationships between these factors [75]. Li et al. also used ISM to assess the risks of India's thermal power plants [76].

The advantage of ISM is that it requires fewer questionnaires than other methods, such as structural equation modeling and the Delphi technique, and is able to extract a clear structural view from unstructured models $[77,78]$. However, in ISM, only the existence of an influencing relationship between two factors is investigated; if there is a relationship between factors, it is denoted by " 1 ", otherwise, it is denoted by " 0 " [78]. However, the association between the factors is fuzzy and cannot be easily divided into "related" or "unrelated" [79]. To solve this problem, many scholars have applied fuzzy set theory to ISM, in order to consider the degree of influence between factors, in an approach called fuzzy interpretative structural modeling (FISM) [78,80,81].

As described in the previous section, this study uses the triangular fuzzy number method integrated with ISM in this paper. The process is as follows.

First, the Boolean operation of the skeleton matrix and the identity matrix is performed, where the operation rules are as follows:

$$
0+0=0,0+1=1,1+1=1,0 * 0=0,0 * 1=0,1 * 0=0, \text { and } 1 * 1=1
$$

Meanwhile, it must follow the shift law characteristic of matrix operations; that is, when $a$ directly reaches $b$ through a path of length 1 and $b$ directly reaches $c$ through a path of length 1 , then $a$ must reach $c$ through a path of length 2 . 
Then, the reachability matrix $\mathrm{R}$ can be obtained through a Boolean operation of the skeleton matrix $\mathrm{S}$. When all products are equal, the reachability matrix $\mathrm{R}$ can be obtained.

$$
(S+I) \neq(S+I)^{2} \neq \cdots \neq(S+I)^{r-1} \neq(S+I)^{k}=(S+I)^{k+1}=R
$$

Lastly, based on the reachability matrix, the risk factors of failure are rearranged according to the value of driving power and the reconstituted reachability matrix $R^{*}$ is obtained.

\subsection{Step 5: Using ISM to Hierarchize Risk Factors}

According to the interaction relation of failure risk factors, the hierarchical results can be obtained by referring to the values of elements in the reconstructed reachability matrix. Connecting all the factors with arrow lines, the ISM diagram of the interaction of failure risk factors will be achieved.

\subsection{Step 6: Using MICMAC to Classify Risk Factors}

After ISM, MICMAC (matrix impact cross-reference multiplication applied to a classification) is often used as a complementary method to analyze the driver power and dependence power of the risks [82]. The driver power of a risk means the total number of risks it can influence, whereas the dependence power of a risk means the total number of risks which can influence it [83]. According to the driver and dependence powers of the factors, they can be divided into four groups: autonomous, dependent, linkage, and independent. Issues having weak driving and dependence powers are in the autonomous group, whereas issues having strong dependent and driving powers are in the linkage group [78]. Issues which have strong driving and weak dependence powers are known as independent issues, whereas issues which have strong dependence power and weak driving power are known as dependent issues [78].

Many scholars have applied ISM-MICMAC to PPP risk analysis. Iyer et al. analyzed the hierarchical structure of PPP risks of Indian roads for the first time by combining ISM and MICMAC [19]. Han et al. used ISM-MICMAC to analyze PPP risks in brownfield remediation projects in China [83]. Li and Wang used a fuzzy analytic network process and ISM-MICMAC for PPP risk assessment [18].

Although these analyses based on ISM-MICMAC have achieved some beneficial results, they have all ignored the notion that the impact degree of PPP risks is vague and cannot be judged simply by the impact or no impact. Based on this, FISM is adopted in this paper to consider the fuzziness of the relationships between PPP risks, replacing the traditional " 0 or 1 " judgment with a fuzzy number, in order to reduce the subjectivity of expert judgment.

In this study, the categories of risk factors are determined by FISM integrated MICMAC method. Based on the reconstituted reachability matrix $\mathrm{R}^{\wedge *}$, each factor is plotted as a point in the four quadrants of the rectangular coordinate system, according to the driving power and dependency power of each failure risk factor, and the key risk factors are classified.

\section{Results and Findings}

By following Step 1 in Section 3, a literature review and case study analysis was conducted to elicit the preliminary list of risk factors. A total of 29 preliminary risk factors were extracted to the preliminary risk list.

To mitigate the deficiency of the literature review and case study, we invited five experts for two rounds of interviews to validate the preliminary list. We chose experts who had sufficient time available for being interviewed and for summarizing these risk factors. Other experts that were considered, but who were too busy, were excluded from the risk identification stage. These experts included one lawyer involved in PPP consulting, one manager of a PPP project company, two managers of a PPP consulting company and one PPP research scholar, all of whom have participated in the management of PPP projects and have a rich knowledge of PPP theory and practice. 
After the first round of interviews, based on the opinions of various experts and further confirmation in the second round of interviews, the risk factors that led to the failure of PPP projects were summarized into 20 risks (see Table 3). The literature and case sources of these 20 risks can be seen in Tables 4 and 5 .

These 20 risks are: government decision-making approval risk (R1); policy and regulatory change risk (R2); government credit risk (R3); government regulatory risk (R4); planning and design risk (R5); bidding risk (R6); contract risk (R7); facility matching risk (R8); financing risk (R9); economic risk (R10); project change risk (R11); construction risk (R12); project income risk (R13); parallel project competitive risk (R14); operation and maintenance risk (R15); force majeure risk (R16); public opposition risk (R17); organizational coordination risk (R18); environmental risk (R19); and project company violates laws and regulations (R20).

In this study, in addition to the five original experts who participated in expert interviews, another 10 experts were invited to conduct a questionnaire survey on the relationship between PPP risk factors by means of e-mail, WeChat and on-site survey (see Appendix B). All 15 experts have rich knowledge of PPP theory and practice, and their background information is shown in the Table 6. Through comparative analysis of questionnaire data, the high impact of other risks on the force majeure risk of R16 was taken as an invalid judgment standard to eliminate the invalid questionnaires. In total, there were 9 valid questionnaires.

Table 3. Preliminary risk list and final risk list.

\begin{tabular}{|c|c|}
\hline Preliminary Risk List (29 Risks) & Final Risk List (20 Risks) \\
\hline $\begin{array}{l}\text { Lengthy government decision-making and approval } \\
\text { Lack of PPP co-operation experience }\end{array}$ & Government decision-making approval risk (R1) \\
\hline Policy and regulatory change risk & Policy and regulatory change risk (R2) \\
\hline Government official corruption & Government credit risk (R3) \\
\hline $\begin{array}{l}\text { Government intervention } \\
\text { Government regulatory risk }\end{array}$ & Government regulatory risk (R4) \\
\hline Planning and design risk & Planning and design risk (R5) \\
\hline Bidding risk & Bidding risk (R6) \\
\hline Imperfect price adjustment mechanism & \\
\hline $\begin{array}{l}\text { Unreasonable profit distribution } \\
\text { Imperfect dispute settlement mechanism } \\
\text { Incomplete contract risk }\end{array}$ & Contract risk (R7) \\
\hline Facility matching risk & Facility matching risk (R8) \\
\hline Financing risk & Financing risk (R9) \\
\hline Economic risk & Economic risk (R10) \\
\hline Project change risk & Project change risk (R11) \\
\hline Construction cost overruns & \\
\hline $\begin{array}{l}\text { Construction quality risk } \\
\text { Construction schedule risk } \\
\text { Completion risks }\end{array}$ & Construction risk (R12) \\
\hline $\begin{array}{l}\text { Changes in market demand } \\
\text { Insufficient expense payment }\end{array}$ & Project income risk (R13) \\
\hline Parallel project competitive risk & Parallel project competitive risk (R14) \\
\hline Operation and maintenance risk & Operation and maintenance risk (R15) \\
\hline Force majeure risk & Force majeure risk (R16) \\
\hline Public opposition risk & Public opposition risk (R17) \\
\hline Organizational coordination risk & Organizational coordination risk (R18) \\
\hline Environmental risk & Environmental risk (R19) \\
\hline Project company violates laws and regulations & Project company violates laws and regulations (R20) \\
\hline
\end{tabular}


Table 4. Literature statistics for PPP risk factors.

\begin{tabular}{|c|c|c|c|c|c|c|c|c|c|c|c|c|c|c|c|c|}
\hline Risk Factors & $1[38]$ & $2[83]$ & $3[84]$ & $4[18]$ & 5 [39] & $6[85]$ & $7[86]$ & $8[34]$ & 9 [29] & $10[26]$ & 11 [12] & 12 [11] & 13 [17] & 14 [87] & $15[27]$ & $16[44]$ \\
\hline $\begin{array}{l}\text { Government decision-making } \\
\text { approval risk (R1) }\end{array}$ & $\mathrm{O}$ & $\mathrm{O}$ & $\mathrm{O}$ & $\mathrm{O}$ & $\mathrm{O}$ & $\mathrm{O}$ & $\mathrm{O}$ & $\mathrm{O}$ & $\mathrm{O}$ & $\mathrm{O}$ & $\mathrm{O}$ & $\mathrm{O}$ & $\mathrm{O}$ & $\mathrm{O}$ & $\mathrm{O}$ & $\mathrm{O}$ \\
\hline $\begin{array}{l}\text { Policy and regulatory change } \\
\text { risk (R2) }\end{array}$ & $\mathrm{O}$ & $\mathrm{O}$ & $\mathrm{O}$ & $\mathrm{O}$ & $\mathrm{O}$ & $\mathrm{O}$ & $\mathrm{O}$ & $\mathrm{O}$ & $\mathrm{O}$ & $\mathrm{O}$ & $\mathrm{O}$ & $\mathrm{O}$ & $\mathrm{O}$ & $\mathrm{O}$ & $\mathrm{O}$ & $\mathrm{O}$ \\
\hline Government credit risk (R3) & $\mathrm{O}$ & $\mathrm{O}$ & $\mathrm{O}$ & $\mathrm{O}$ & $\mathrm{O}$ & $\mathrm{O}$ & $\mathrm{O}$ & $\mathrm{O}$ & $\mathrm{O}$ & $\mathrm{O}$ & $\mathrm{O}$ & $\mathrm{O}$ & $\mathrm{O}$ & $\mathrm{O}$ & $\mathrm{O}$ & $\mathrm{O}$ \\
\hline Government regulatory risk ( $\mathrm{R} 4)$ & $\mathrm{O}$ & $\mathrm{O}$ & $\mathrm{O}$ & $\mathrm{O}$ & $\mathrm{O}$ & $\mathrm{O}$ & $\mathrm{O}$ & $\mathrm{O}$ & $\mathrm{O}$ & $\mathrm{O}$ & $\mathrm{O}$ & $\mathrm{O}$ & $\mathrm{O}$ & $\mathrm{O}$ & $\mathrm{O}$ & $\mathrm{O}$ \\
\hline Planning and design risk (R5) & $\mathrm{O}$ & $\mathrm{O}$ & $\mathrm{O}$ & $\mathrm{O}$ & $\mathrm{O}$ & $\mathrm{O}$ & $\mathrm{O}$ & $\mathrm{O}$ & $\mathrm{O}$ & $\mathrm{O}$ & $\mathrm{O}$ & $\mathrm{O}$ & $\mathrm{O}$ & $\mathrm{O}$ & $\mathrm{O}$ & $\mathrm{O}$ \\
\hline Bidding risk (R6) & & $\mathrm{O}$ & $\mathrm{O}$ & $\mathrm{O}$ & & $\mathrm{O}$ & & & & $\mathrm{O}$ & & $\mathrm{O}$ & $\mathrm{O}$ & $\mathrm{O}$ & & $\mathrm{O}$ \\
\hline Contract risk (R7) & & $\mathrm{O}$ & $\mathrm{O}$ & $\mathrm{O}$ & $\mathrm{O}$ & $\mathrm{O}$ & $\mathrm{O}$ & $\mathrm{O}$ & $\mathrm{O}$ & $\mathrm{O}$ & $\mathrm{O}$ & $\mathrm{O}$ & $\mathrm{O}$ & $\mathrm{O}$ & $\mathrm{O}$ & $\mathrm{O}$ \\
\hline Facility matching risk (R8) & $\mathrm{O}$ & $\mathrm{O}$ & & & $\mathrm{O}$ & & & & $\mathrm{O}$ & & & $\mathrm{O}$ & $\mathrm{O}$ & $\mathrm{O}$ & $\mathrm{O}$ & $\mathrm{O}$ \\
\hline Financing risk (R9) & $\mathrm{O}$ & $\mathrm{O}$ & $\mathrm{O}$ & $\mathrm{O}$ & $\mathrm{O}$ & $\mathrm{O}$ & $\mathrm{O}$ & $\mathrm{O}$ & $\mathrm{O}$ & $\mathrm{O}$ & $\mathrm{O}$ & $\mathrm{O}$ & $\mathrm{O}$ & $\mathrm{O}$ & & $\mathrm{O}$ \\
\hline Economic risk (R10) & $\mathrm{O}$ & $\mathrm{O}$ & $\mathrm{O}$ & $\mathrm{O}$ & $\mathrm{O}$ & $\mathrm{O}$ & $\mathrm{O}$ & $\mathrm{O}$ & $\mathrm{O}$ & $\mathrm{O}$ & $\mathrm{O}$ & $\mathrm{O}$ & $\mathrm{O}$ & $\mathrm{O}$ & & $\mathrm{O}$ \\
\hline Project change risk (R11) & & $\mathrm{O}$ & $\mathrm{O}$ & $\mathrm{O}$ & $\mathrm{O}$ & $\mathrm{O}$ & $\mathrm{O}$ & $\mathrm{O}$ & $\mathrm{O}$ & $\mathrm{O}$ & & $\mathrm{O}$ & $\mathrm{O}$ & $\mathrm{O}$ & $\mathrm{O}$ & $\mathrm{O}$ \\
\hline Construction risk (R12) & $\mathrm{O}$ & $\mathrm{O}$ & $\mathrm{O}$ & $\mathrm{O}$ & $\mathrm{O}$ & $\mathrm{O}$ & $\mathrm{O}$ & $\mathrm{O}$ & $\mathrm{O}$ & $\mathrm{O}$ & $\mathrm{O}$ & $\mathrm{O}$ & $\mathrm{O}$ & $\mathrm{O}$ & $\mathrm{O}$ & $\mathrm{O}$ \\
\hline Project income risk (R13) & $\mathrm{O}$ & $\mathrm{O}$ & $\mathrm{O}$ & $\mathrm{O}$ & $\mathrm{O}$ & $\mathrm{O}$ & $\mathrm{O}$ & $\mathrm{O}$ & $\mathrm{O}$ & $\mathrm{O}$ & $\mathrm{O}$ & $\mathrm{O}$ & $\mathrm{O}$ & $\mathrm{O}$ & $\mathrm{O}$ & $\mathrm{O}$ \\
\hline $\begin{array}{l}\text { Parallel project competitive } \\
\text { risk (R14) }\end{array}$ & & & & $\mathrm{O}$ & $\mathrm{O}$ & $\mathrm{O}$ & $\mathrm{O}$ & & $\mathrm{O}$ & & & $\mathrm{O}$ & $\mathrm{O}$ & $\mathrm{O}$ & $\mathrm{O}$ & \\
\hline $\begin{array}{l}\text { Operation and maintenance } \\
\text { risk (R15) }\end{array}$ & $\mathrm{O}$ & $\mathrm{O}$ & $\mathrm{O}$ & $\mathrm{O}$ & $\mathrm{O}$ & $\mathrm{O}$ & $\mathrm{O}$ & $\mathrm{O}$ & $\mathrm{O}$ & $\mathrm{O}$ & $\mathrm{O}$ & $\mathrm{O}$ & $\mathrm{O}$ & $\mathrm{O}$ & $\mathrm{O}$ & $\mathrm{O}$ \\
\hline Force majeure risk (R16) & $\mathrm{O}$ & $\mathrm{O}$ & $\mathrm{O}$ & $\mathrm{O}$ & $\mathrm{O}$ & & $\mathrm{O}$ & $\mathrm{O}$ & $\mathrm{O}$ & $\mathrm{O}$ & $\mathrm{O}$ & $\mathrm{O}$ & $\mathrm{O}$ & $\mathrm{O}$ & $\mathrm{O}$ & $\mathrm{O}$ \\
\hline Public opposition risk (R17) & & $\mathrm{O}$ & $\mathrm{O}$ & $\mathrm{O}$ & $\mathrm{O}$ & & $\mathrm{O}$ & $\mathrm{O}$ & $\mathrm{O}$ & $\mathrm{O}$ & & $\mathrm{O}$ & $\mathrm{O}$ & $\mathrm{O}$ & $\mathrm{O}$ & $\mathrm{O}$ \\
\hline $\begin{array}{c}\text { Organizational coordination } \\
\text { risk (R18) }\end{array}$ & & $\mathrm{O}$ & $\mathrm{O}$ & $\mathrm{O}$ & $\mathrm{O}$ & $\mathrm{O}$ & $\mathrm{O}$ & $\mathrm{O}$ & $\mathrm{O}$ & $\mathrm{O}$ & & $\mathrm{O}$ & $\mathrm{O}$ & $\mathrm{O}$ & & $\mathrm{O}$ \\
\hline Environmental risk (R19) & & $\mathrm{O}$ & $\mathrm{O}$ & $\mathrm{O}$ & $\mathrm{O}$ & & $\mathrm{O}$ & $\mathrm{O}$ & $\mathrm{O}$ & $\mathrm{O}$ & $\mathrm{O}$ & $\mathrm{O}$ & $\mathrm{O}$ & $\mathrm{O}$ & $\mathrm{O}$ & $\mathrm{O}$ \\
\hline $\begin{array}{l}\text { Project company violates laws } \\
\text { and regulations (R20) }\end{array}$ & & $\mathrm{O}$ & $\mathrm{O}$ & $\mathrm{O}$ & $\mathrm{O}$ & $\mathrm{O}$ & & & $\mathrm{O}$ & $\mathrm{O}$ & & & & & & \\
\hline
\end{tabular}


Table 5. Case statistics for PPP risk factors.

\begin{tabular}{|c|c|c|c|c|c|c|c|c|c|c|c|c|c|c|c|c|c|c|c|c|c|c|c|c|c|c|c|c|}
\hline $\begin{array}{l}\text { Cases } \\
\text { Risk Factors }\end{array}$ & 1 & 2 & 3 & 4 & 5 & 6 & 7 & 8 & 9 & 10 & 11 & 12 & 13 & 14 & 15 & 16 & 17 & 18 & 19 & 20 & 21 & 22 & 23 & 24 & 25 & 26 & 27 & 28 \\
\hline $\mathrm{R} 1$ & & $\sqrt{ }$ & $\sqrt{ }$ & $\sqrt{ }$ & $\sqrt{ }$ & $\sqrt{ }$ & $\sqrt{ }$ & $\sqrt{ }$ & & & & & & & & & & & & & & $\sqrt{ }$ & & & & & & \\
\hline R2 & $\sqrt{ }$ & & & & $\sqrt{ }$ & & & $\sqrt{ }$ & & & & & & $\sqrt{ }$ & & & & $\sqrt{ }$ & & & & & & $\sqrt{ }$ & & & $\sqrt{ }$ & \\
\hline R3 & $\sqrt{ }$ & & & & $\sqrt{ }$ & $\sqrt{ }$ & $\sqrt{ }$ & & $\sqrt{ }$ & $\sqrt{ }$ & & $\sqrt{ }$ & & $\sqrt{ }$ & $\sqrt{ }$ & $\sqrt{ }$ & $\sqrt{ }$ & $\sqrt{ }$ & & $\sqrt{ }$ & $\sqrt{ }$ & & $\sqrt{ }$ & & & $\sqrt{ }$ & & $\sqrt{ }$ \\
\hline R4 & $\sqrt{ }$ & $\sqrt{ }$ & & & & & & & & $\sqrt{ }$ & $\sqrt{ }$ & $\sqrt{ }$ & & & & $\sqrt{ }$ & & & & $\sqrt{ }$ & & & & & & & $\sqrt{ }$ & \\
\hline R5 & & & & & & & & $\sqrt{ }$ & & & & $\sqrt{ }$ & $\sqrt{ }$ & & & & $\sqrt{ }$ & & $\sqrt{ }$ & $\sqrt{ }$ & $\sqrt{ }$ & $\sqrt{ }$ & & & & $\sqrt{ }$ & & $\sqrt{ }$ \\
\hline R6 & & & & & & & & & & & $\sqrt{ }$ & & & & & & & & & & & & & $\sqrt{ }$ & $\sqrt{ }$ & & $\sqrt{ }$ & $\sqrt{ }$ \\
\hline R7 & & $\sqrt{ }$ & $\sqrt{ }$ & & $\sqrt{ }$ & $\sqrt{ }$ & $\sqrt{ }$ & $\sqrt{ }$ & $\sqrt{ }$ & $\sqrt{ }$ & & & $\sqrt{ }$ & $\sqrt{ }$ & & & & $\sqrt{ }$ & & & & & $\sqrt{ }$ & & & $\sqrt{ }$ & & \\
\hline R8 & & & $\sqrt{ }$ & & & & & & & & & & & & & & & & $\sqrt{ }$ & & & & $\sqrt{ }$ & & & & & \\
\hline R9 & & & & $\sqrt{ }$ & & & & & & & & & & & & & & & & & & & & $\sqrt{ }$ & & & $\sqrt{ }$ & \\
\hline R10 & & & & & & & & & & & & & $\sqrt{ }$ & & & & & & & & & & & & & & & \\
\hline R11 & & & & $\sqrt{ }$ & & & & & & & & & & & $\sqrt{ }$ & & & & & & $\sqrt{ }$ & & & & & & & $\sqrt{ }$ \\
\hline R12 & & & & $\sqrt{ }$ & & & & & $\sqrt{ }$ & & & & $\sqrt{ }$ & & $\sqrt{ }$ & $\sqrt{ }$ & $\sqrt{ }$ & & & & & & & $\sqrt{ }$ & $\sqrt{ }$ & & & $\sqrt{ }$ \\
\hline R13 & $\sqrt{ }$ & & $\sqrt{ }$ & $\sqrt{ }$ & & $\sqrt{ }$ & $\sqrt{ }$ & $\sqrt{ }$ & & & $\sqrt{ }$ & $\sqrt{ }$ & $\sqrt{ }$ & $\sqrt{ }$ & $\sqrt{ }$ & $\sqrt{ }$ & & $\sqrt{ }$ & $\sqrt{ }$ & $\sqrt{ }$ & $\sqrt{ }$ & & $\sqrt{ }$ & & $\sqrt{ }$ & & & \\
\hline R14 & & & & & & & & & & & & & $\sqrt{ }$ & $\sqrt{ }$ & $\sqrt{ }$ & $\sqrt{ }$ & & & & & & & & & & $\sqrt{ }$ & & \\
\hline R15 & & $\sqrt{ }$ & & & & & & & & $\sqrt{ }$ & & & & & & & & & & & & & & & $\sqrt{ }$ & & & \\
\hline R16 & & & & & & & & & & & & & & & & & $\sqrt{ }$ & & & & & & & & & & & \\
\hline R17 & & $\sqrt{ }$ & & $\sqrt{ }$ & & & & & & $\sqrt{ }$ & & & & & & & $\sqrt{ }$ & & & & & $\sqrt{ }$ & & & $\sqrt{ }$ & $\sqrt{ }$ & & \\
\hline R18 & & & & & & & & & & & $\sqrt{ }$ & & & & & & $\sqrt{ }$ & & & & & & & $\sqrt{ }$ & $\sqrt{ }$ & & $\sqrt{ }$ & \\
\hline R19 & $\sqrt{ }$ & $\sqrt{ }$ & & & & & & & & $\sqrt{ }$ & 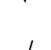 & $\sqrt{ }$ & & & & & & & & $\sqrt{ }$ & & $\sqrt{ }$ & $\sqrt{ }$ & & & & & \\
\hline R20 & $\sqrt{ }$ & $\sqrt{ }$ & & & & & & & & $\sqrt{ }$ & $\sqrt{ }$ & & & & & & & & & & $\sqrt{ }$ & & & & & & & \\
\hline
\end{tabular}


Table 6. Background information of 15 experts.

\begin{tabular}{ccccc}
\hline No. & Education Profile & Work Experience & $\begin{array}{c}\text { Number of PPP } \\
\text { Participated }\end{array}$ & Company \\
\hline 1 & PhD & 6 to 10 years & Three & University \\
2 & PhD & Under 5 years & One & University \\
3 & Master & 6 to 10 years & Two & Government \\
4 & Master & 11 to 15 years & More than 4 & Consulting \\
5 & Master & 6 to 10 years & Three & Consulting \\
6 & Master & Under 5 years & Two & Consulting \\
7 & Bachelor & 11 to 15 years & Three & Consulting \\
8 & Master & 6 to 10 years & One & Private \\
9 & Master & 6 to 10 years & One & Private \\
10 & Master & 11 to 15 years & Two & Private \\
11 & Master & 6 to 10 years & One & Private \\
12 & Master & 6 to 10 years & Two & Private \\
13 & Bachelor & More than 16 years & Two & Private \\
14 & Bachelor & More than 16 years & Two & Private \\
15 & Bachelor & 11 to 15 years & Two & Private \\
\hline
\end{tabular}

The triangular fuzzy number of 9 valid questionnaires were filled in the triangular fuzzy relation matrix. Then, we converted triangular fuzzy relation matrix to fuzzy direct relation matrix through converting fuzzy data into crisp scores (CFCS) method. The final fuzzy direct relation matrix is shown in Table 7 .

Table 7. Fuzzy direct relation matrix of the PPP key risk factors.

\begin{tabular}{|c|c|c|c|c|c|c|c|c|c|c|c|c|c|c|c|c|c|c|c|c|}
\hline Risk & R1 & R2 & R3 & R4 & R5 & R6 & R7 & R8 & R9 & R10 & R11 & R12 & R13 & R14 & R15 & R16 & R17 & R18 & R19 & R20 \\
\hline R1 & 0 & & & & & & & .36 & & & & & & 0.49 & & 0.00 & 0.39 & 0.38 & 0.28 & 0.13 \\
\hline $\mathrm{R} 2$ & 0.38 & 0 & 0.54 & 0.40 & 15 & 0.23 & 0.54 & 0.12 & 0.43 & 0.41 & 0.63 & 0.38 & 0.35 & 0.24 & 0.33 & 0.00 & 0.19 & 0.14 & 0.46 & 0.20 \\
\hline R3 & 0.40 & 0.25 & 0 & 0.38 & 0.24 & 0.30 & & 0.37 & 0.39 & 0.31 & 0.45 & & 0.45 & 0.65 & & 0.08 & 0.25 & 0.35 & .15 & 0.20 \\
\hline $\mathrm{R} 4$ & 0.29 & 0.05 & 0.40 & 0 & 0.33 & 0.53 & 0.31 & 0.41 & 0.35 & 0.28 & 0.30 & 0.60 & 0.35 & 0.31 & 0.33 & 0.02 & 0.46 & 0.37 & 0.52 & 0.49 \\
\hline R5 & 0.18 & 0.04 & 0.10 & 0.04 & 0 & 0.28 & 0.35 & 0.25 & 0.32 & 0.18 & 0.74 & 0.38 & 0.57 & 0.31 & 0.38 & 0.00 & 0.51 & 0.09 & 0.45 & 0.02 \\
\hline R6 & 0.10 & 0.15 & 0.19 & 0.23 & 0.17 & 0 & 0.43 & 0.23 & 0.31 & 0.22 & 0.37 & 0.45 & 0.60 & 0.07 & 0.57 & 0.02 & 0.15 & 0.32 & 0.19 & 0.45 \\
\hline R7 & 0.12 & 0.15 & 0.30 & 0.35 & 0.15 & 0.07 & 0 & 0.27 & 0.19 & 0.28 & 0.43 & 0.46 & 0.45 & 0.18 & 0.40 & 0.12 & 0.19 & 0.40 & 0.25 & 0.38 \\
\hline R8 & 0.17 & 0.05 & 0.17 & 0.07 & 0.19 & 0.15 & 0.33 & 0 & 0.24 & 0.12 & 0.33 & 0.60 & 0.38 & 0.08 & 0.49 & 0.00 & 0.09 & 0.10 & 0.15 & 0.22 \\
\hline R9 & 0.10 & 0.10 & 0.12 & 0.07 & 0.10 & 0.12 & 0.38 & 0.10 & 0 & 0.33 & 0.22 & 0.50 & 0.53 & 0.04 & 0.47 & 0.00 & 0.07 & 0.15 & 0.12 & 0.25 \\
\hline R10 & 0.25 & 0.32 & 0.27 & 0.14 & 0.04 & 0.17 & 0.31 & 0.18 & 0.59 & 0 & 0.16 & 0.35 & 0.53 & 0.10 & 0.35 & 0.00 & 0.19 & 0.12 & 0.12 & 0.41 \\
\hline R11 & 0.15 & 0.12 & 0.38 & 0.28 & 0.25 & 0.09 & 0.41 & 0.30 & 0.39 & 0.02 & 0 & 0.71 & 0.60 & 0.07 & 0.52 & 0.02 & 0.11 & 0.27 & 0.24 & 0.27 \\
\hline R12 & 0.04 & 0.07 & 0.27 & 0.25 & 0.16 & 0.07 & 0.43 & 0.15 & 0.33 & 0.02 & 0.45 & 0 & 0.65 & 0.07 & 0.35 & 0.13 & 0.35 & 0.20 & 0.35 & 0.33 \\
\hline R13 & 0.16 & 0.15 & 0.44 & 0.22 & 0.10 & 0.10 & 0.44 & 0.12 & 0.60 & 0.10 & 0.43 & 0.32 & 0 & 0.27 & 0.46 & 0.00 & 0.27 & 0.17 & 0.10 & 0.36 \\
\hline R14 & 0.12 & 0.12 & 0.57 & 0.20 & 0.15 & 0.15 & 0.52 & 0.12 & 0.31 & 0.07 & 0.36 & 0.26 & 0.52 & 0 & 0.24 & 0.00 & 0.14 & 0.17 & 0.04 & 0.41 \\
\hline R15 & 0.07 & 0.12 & 0.30 & 0.23 & 0.17 & 0.07 & 0.38 & 0.17 & 0.22 & 0.10 & 0.28 & 0.04 & 0.80 & 0.10 & 0 & 0.00 & 0.37 & 0.22 & 0.46 & 0.32 \\
\hline R16 & 0.12 & 0.15 & 0.33 & 0.22 & 0.10 & 0.10 & 0.33 & 0.22 & 0.38 & 0.24 & 0.52 & 0.43 & 0.47 & 0.04 & 0.55 & 0 & 0.12 & 0.17 & 0.29 & 0.22 \\
\hline R17 & & 0.22 & 0.46 & 0.33 & 0.27 & 0.09 & & 0.19 & & & & 0.45 & & 0.17 & & 0.07 & 0 & 0.17 & 0.20 & 0.17 \\
\hline R18 & 0.33 & 0.09 & 0.32 & 0.43 & 0.33 & 0.32 & 0.46 & 0.23 & 0.35 & 0.04 & 0.33 & 0.57 & 0.49 & 0.17 & 0.52 & 0.02 & 0.25 & 0 & 0.15 & 0.41 \\
\hline R19 & 0.22 & 0.32 & 0.33 & 0.33 & 0.30 & 0.10 & 0.35 & 0.20 & 0.24 & 0.04 & 0.51 & 0.51 & 0.63 & 0.07 & 0.54 & 0.07 & 0.77 & 0.12 & 0 & 0.28 \\
\hline R20 & 0.04 & 0.11 & 0.35 & 0.28 & 0.18 & 0.23 & 0.55 & 0.17 & 0.38 & 0.04 & 0.41 & 0.54 & 0.57 & 0.12 & 0.48 & 0.00 & 0.60 & 0.30 & 0.60 & 0 \\
\hline
\end{tabular}

After obtaining the fuzzy direct relation matrix, the intercept coefficient $\alpha$ was adopted to convert the fuzzy direct relation matrix into a skeleton matrix, which was directly used for Boolean operation, as shown in Table 8. 
Table 8. Skeleton matrix of the PPP key risk factors.

\begin{tabular}{ccccccccccccccccccccc}
\hline Risk & R1 & R2 & R3 & R4 & R5 & R6 & R7 & R8 & R9 & R10 & R11 & $\mathbf{R 1 2}$ & $\mathbf{R 1 3}$ & $\mathbf{R 1 4}$ & $\mathbf{R 1 5}$ & $\mathbf{R 1 6}$ & $\mathbf{R} 17$ & R18 & R19 & R20 \\
\hline R1 & 1 & 0 & 1 & 0 & 1 & 0 & 0 & 0 & 0 & 0 & 0 & 0 & 0 & 0 & 0 & 0 & 0 & 0 & 0 & 0 \\
R2 & 0 & 1 & 1 & 0 & 0 & 0 & 1 & 0 & 0 & 0 & 1 & 0 & 0 & 0 & 0 & 0 & 0 & 0 & 0 & 0 \\
R3 & 0 & 0 & 1 & 0 & 0 & 0 & 0 & 0 & 0 & 0 & 0 & 0 & 0 & 1 & 0 & 0 & 0 & 0 & 0 & 0 \\
R4 & 0 & 0 & 0 & 1 & 0 & 1 & 0 & 0 & 0 & 0 & 0 & 1 & 0 & 0 & 0 & 0 & 0 & 0 & 1 & 0 \\
R5 & 0 & 0 & 0 & 0 & 1 & 0 & 0 & 0 & 0 & 0 & 1 & 0 & 1 & 0 & 0 & 0 & 1 & 0 & 0 & 0 \\
R6 & 0 & 0 & 0 & 0 & 0 & 1 & 0 & 0 & 0 & 0 & 0 & 0 & 1 & 0 & 1 & 0 & 0 & 0 & 0 & 0 \\
R7 & 0 & 0 & 0 & 0 & 0 & 0 & 1 & 0 & 0 & 0 & 0 & 0 & 0 & 0 & 0 & 0 & 0 & 0 & 0 & 0 \\
R8 & 0 & 0 & 0 & 0 & 0 & 0 & 0 & 1 & 0 & 0 & 0 & 1 & 0 & 0 & 0 & 0 & 0 & 0 & 0 & 0 \\
R9 & 0 & 0 & 0 & 0 & 0 & 0 & 0 & 0 & 1 & 0 & 0 & 1 & 1 & 0 & 0 & 0 & 0 & 0 & 0 & 0 \\
R10 & 0 & 0 & 0 & 0 & 0 & 0 & 0 & 0 & 1 & 1 & 0 & 0 & 1 & 0 & 0 & 0 & 0 & 0 & 0 & 0 \\
R11 & 0 & 0 & 0 & 0 & 0 & 0 & 0 & 0 & 0 & 0 & 1 & 1 & 1 & 0 & 1 & 0 & 0 & 0 & 0 & 0 \\
R12 & 0 & 0 & 0 & 0 & 0 & 0 & 0 & 0 & 0 & 0 & 0 & 1 & 1 & 0 & 0 & 0 & 0 & 0 & 0 & 0 \\
R13 & 0 & 0 & 0 & 0 & 0 & 0 & 0 & 0 & 1 & 0 & 0 & 0 & 1 & 0 & 0 & 0 & 0 & 0 & 0 & 0 \\
R14 & 0 & 0 & 1 & 0 & 0 & 0 & 1 & 0 & 0 & 0 & 0 & 0 & 1 & 1 & 0 & 0 & 0 & 0 & 0 & 0 \\
R15 & 0 & 0 & 0 & 0 & 0 & 0 & 0 & 0 & 0 & 0 & 0 & 0 & 1 & 0 & 1 & 0 & 0 & 0 & 0 & 0 \\
R16 & 0 & 0 & 0 & 0 & 0 & 0 & 0 & 0 & 0 & 0 & 1 & 0 & 0 & 0 & 1 & 1 & 0 & 0 & 0 & 0 \\
R17 & 1 & 0 & 0 & 0 & 0 & 0 & 0 & 0 & 0 & 0 & 1 & 0 & 0 & 0 & 0 & 0 & 1 & 0 & 0 & 0 \\
R18 & 0 & 0 & 0 & 0 & 0 & 0 & 0 & 0 & 0 & 0 & 0 & 1 & 0 & 0 & 1 & 0 & 0 & 1 & 0 & 0 \\
R19 & 0 & 0 & 0 & 0 & 0 & 0 & 0 & 0 & 0 & 0 & 1 & 1 & 1 & 0 & 1 & 0 & 1 & 0 & 1 & 0 \\
R20 & 0 & 0 & 0 & 0 & 0 & 0 & 1 & 0 & 0 & 0 & 0 & 1 & 1 & 0 & 0 & 0 & 1 & 0 & 1 & 1 \\
\hline
\end{tabular}

Reachability matrix was obtained after Boolean operation of the skeleton matrix, and on the basis of the reachability matrix, all failure risk factors were rearranged according to the value of driving power to get the reconfigurable reachability matrix (see in Table 9). In order to reduce the amount of calculation, we used Python programming in the operation process.

Table 9. Reconfigured reachability matrix of the PPP key risk factors.

\begin{tabular}{cccccccccccccccccccccc}
\hline Risk & R7 & R9 & R12 & R13 & R8 & R10 & R15 & R6 & R11 & R18 & R3 & R14 & R16 & R2 & R1 & R5 & R17 & R19 & R20 & R4 & Dr \\
\hline R7 & 1 & 0 & 0 & 0 & 0 & 0 & 0 & 0 & 0 & 0 & 0 & 0 & 0 & 0 & 0 & 0 & 0 & 0 & 0 & 0 & 1 \\
R9 & 0 & 1 & 1 & 1 & 0 & 0 & 0 & 0 & 0 & 0 & 0 & 0 & 0 & 0 & 0 & 0 & 0 & 0 & 0 & 0 & 3 \\
R12 & 0 & 1 & 1 & 1 & 0 & 0 & 0 & 0 & 0 & 0 & 0 & 0 & 0 & 0 & 0 & 0 & 0 & 0 & 0 & 0 & 3 \\
R13 & 0 & 1 & 1 & 1 & 0 & 0 & 0 & 0 & 0 & 0 & 0 & 0 & 0 & 0 & 0 & 0 & 0 & 0 & 0 & 0 & 3 \\
R8 & 0 & 1 & 1 & 1 & 1 & 0 & 0 & 0 & 0 & 0 & 0 & 0 & 0 & 0 & 0 & 0 & 0 & 0 & 0 & 0 & 4 \\
R10 & 0 & 1 & 1 & 1 & 0 & 1 & 0 & 0 & 0 & 0 & 0 & 0 & 0 & 0 & 0 & 0 & 0 & 0 & 0 & 0 & 4 \\
R15 & 0 & 1 & 1 & 1 & 0 & 0 & 1 & 0 & 0 & 0 & 0 & 0 & 0 & 0 & 0 & 0 & 0 & 0 & 0 & 0 & 4 \\
R6 & 0 & 1 & 1 & 1 & 0 & 0 & 1 & 1 & 0 & 0 & 0 & 0 & 0 & 0 & 0 & 0 & 0 & 0 & 0 & 0 & 5 \\
R11 & 0 & 1 & 1 & 1 & 0 & 0 & 1 & 0 & 1 & 0 & 0 & 0 & 0 & 0 & 0 & 0 & 0 & 0 & 0 & 0 & 5 \\
R18 & 0 & 1 & 1 & 1 & 0 & 0 & 1 & 0 & 0 & 1 & 0 & 0 & 0 & 0 & 0 & 0 & 0 & 0 & 0 & 0 & 5 \\
R3 & 1 & 1 & 1 & 1 & 0 & 0 & 0 & 0 & 0 & 0 & 1 & 1 & 0 & 0 & 0 & 0 & 0 & 0 & 0 & 0 & 6 \\
R14 & 1 & 1 & 1 & 1 & 0 & 0 & 0 & 0 & 0 & 0 & 1 & 1 & 0 & 0 & 0 & 0 & 0 & 0 & 0 & 0 & 6 \\
R16 & 0 & 1 & 1 & 1 & 0 & 0 & 1 & 0 & 1 & 0 & 0 & 0 & 1 & 0 & 0 & 0 & 0 & 0 & 0 & 0 & 6 \\
R2 & 1 & 1 & 1 & 1 & 0 & 0 & 1 & 0 & 1 & 0 & 1 & 1 & 0 & 1 & 0 & 0 & 0 & 0 & 0 & 0 & 9 \\
R1 & 1 & 1 & 1 & 1 & 0 & 0 & 1 & 0 & 1 & 0 & 1 & 1 & 0 & 0 & 1 & 1 & 1 & 0 & 0 & 0 & 11 \\
R5 & 1 & 1 & 1 & 1 & 0 & 0 & 1 & 0 & 1 & 0 & 1 & 1 & 0 & 0 & 1 & 1 & 1 & 0 & 0 & 0 & 11 \\
R17 & 1 & 1 & 1 & 1 & 0 & 0 & 1 & 0 & 1 & 0 & 1 & 1 & 0 & 0 & 1 & 1 & 1 & 0 & 0 & 0 & 11 \\
R19 & 1 & 1 & 1 & 1 & 0 & 0 & 1 & 0 & 1 & 0 & 1 & 1 & 0 & 0 & 1 & 1 & 1 & 1 & 0 & 0 & 12 \\
R20 & 1 & 1 & 1 & 1 & 0 & 0 & 1 & 0 & 1 & 0 & 1 & 1 & 0 & 0 & 1 & 1 & 1 & 1 & 1 & 0 & 13 \\
R4 & 1 & 1 & 1 & 1 & 0 & 0 & 1 & 1 & 1 & 0 & 1 & 1 & 0 & 0 & 1 & 1 & 1 & 1 & 0 & 1 & 14 \\
De & 10 & 19 & 19 & 19 & 1 & 1 & 12 & 2 & 9 & 1 & 9 & 9 & 1 & 1 & 6 & 6 & 6 & 3 & 1 & 1 & \\
\hline
\end{tabular}

Risk factors were hierarchized by using the ISM diagram. In the ISM diagram, the classification of a risk factor hierarchy can be determined by the driving power of a risk factor; that is, the risk factor with smaller driving power sits on the higher level in the risk system of ISM diagram, and the risk factor with higher driving power sits on the bottom position in the ISM diagram. We obtained the hierarchical partition results and ISM diagram according to the driving power of each risk in reconfigured reachability matrix. According to the positions of the risk factors in the ISM diagram, they were divided into three areas: upper, middle, and bottom, as shown in Figure 2. In Figure 2, we can see that: 
1. The upper risk factors included R7 (contract risk), R9 (financing risk), R12 (construction risk), and $\mathrm{R} 13$ (project income risk), and there was a strong connection between R9, R12, and R13. These risks directly led to the failure of PPP projects.

2. The middle risk factors included R3 (government credit risk), R11 (project change risk), R14 (parallel project competitive risk), R15 (operation and maintenance risk), R6 (bidding risk), R2 (policies and regulations change risk), R8 (Facility matching risk), R10 (economic risk), R16 (force majeure risk), and R18 (organizational coordination risk).

3. The bottom risk factors included R4 (government regulatory risk), R20 (project company violates laws and regulations), R19 (environmental risk), R17 (public opposition risk), R1 (government decision-making approval risk), and R5 (planning and design risk). It can be considered that these risk factors are the most critical and fundamental in the risk system.

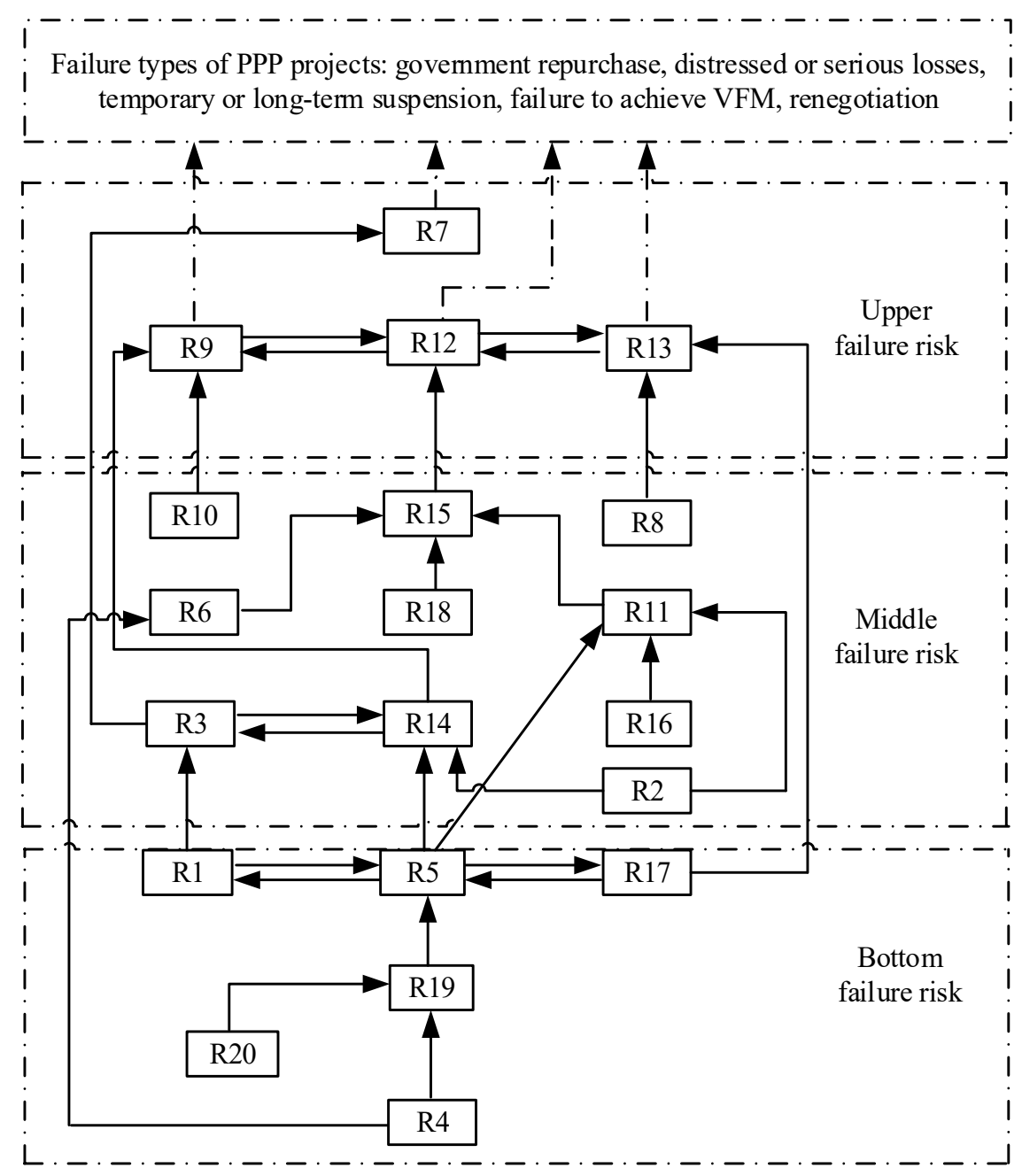

Figure 2. Interpretative structural modeling (ISM) diagram of the PPP key risk factors.

After obtaining the ISM diagram that represents the mutual influence of key risk factors in PPP, it is necessary to refer to the MICMAC method to determine the categories of risk factors, so as to provide corresponding prevention suggestions for different types of risk in a more targeted way.

Based on the reconfigurable reachability matrix, each factor was plotted as a point in the conventional $\mathrm{X}-\mathrm{Y}$ coordinate system according to the values of driving power and dependence power. Thus, the 20 key risk factors were divided into four categories and distributed in four quadrants, as shown in Figure 3. 


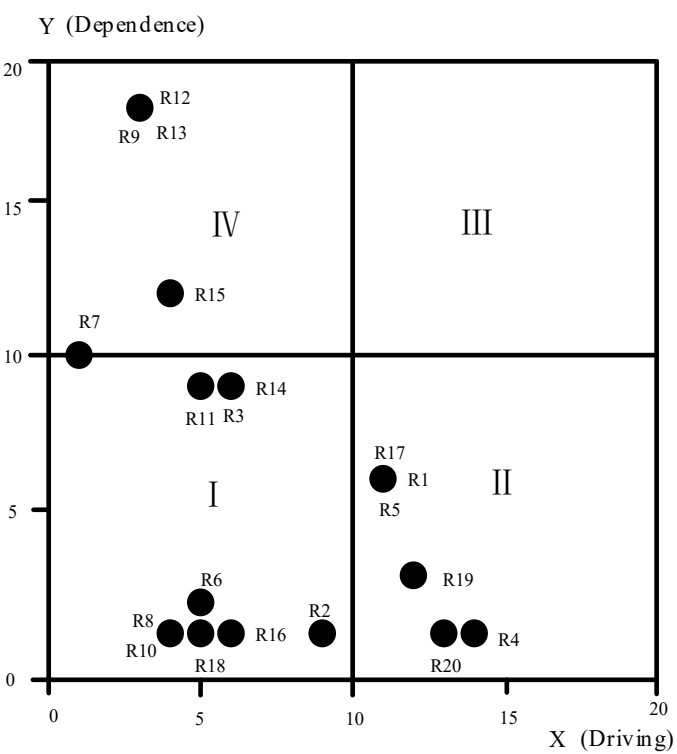

Figure 3. The driving-dependence power diagram of the PPP key risk factors.

Each quadrant contains risk factors as follows: Quadrant I (Autonomous risks) includes: R2 (policies and regulations change risk), R3 (government credit risk), R6 (bidding risk), R8 (facility matching risk), R10 (economic risk), R11 (project change risk), R14 (parallel project competitive risk), R16 (force majeure risk), R18 (organizational coordination risk). The driving power and dependence power of the risk factors are relatively low, which means they are not easily influenced by other risk factors, and also do not easy to lead to the occurrence of other risk factors;

Quadrant II (Dependent risks) includes: R1 (government decision-making approval risk), R5 (planning and design risk), R4 (government regulatory risk), R17 (public opposition risk), R19 (environmental risk), R20 (project company violates laws and regulations). They have a powerful driving power and weak dependence power, which are key risk factors and basic conditions in the system;

Quadrant III (Linkage risks): there is no any risk. It means that there is no risk that is susceptible to, and affects other, risks

Quadrant IV (Independent risks) includes: R7 (contract risk), R9 (financing risk), R12 (construction risk), R13 (project income risk), and R15 (operation and maintenance risk). They all have stronger dependence, meaning that the occurrence of these risk factors is largely due to the change or accumulation of other risk factors.

\section{Analysis and Discussion}

\subsection{Discussion of Method}

In this study, the FISM is adopted to understand the magnitude of the relationships between the risks [80]. This takes the fuzzy of relationships between PPP risks into account, which has a greater advantage than the traditional ISM method. It also helps in identifying the magnitude of cascading relationships between lower-level issues to higher-level issues [78]. In addition, the method of FISM-MICMAC can provide a new risk assessment tool for risk management strategies in the field of construction engineering and management.

FISM combines fuzzy theory and uses fuzzy language conversion to convert the subjective risks that are difficult to quantify, which is caused by too many uncertain factors in PPP, so as to realize the process of risk evaluation from subjective to objective, and to provide a more scientific theoretical basis for risk response. Compared with traditional ISM, FISM has shown greater advantages $[78,80,81,88]$. 
In this study, the reachability matrix is obtained by using the intercept coefficient to fuzzy direct relation matrix. This method shows that risk managers can obtain different reachability matrices through different intercept coefficients, according to their own requirements of the risk correlation degree.

\subsection{Risk Structure Analysis}

(1) The bottom risk factors of Figure 2 can be classified into two categories. One is likely to occur at any stage in the life cycle of a PPP project, including R4 (government regulatory risk), R20 (project company violates laws and regulations), R19 (environmental risk), and R17 (public opposition risk); whereas R1 (government decision-making approval risk) and R5 (planning and design risk) are pre-project risks. Once these occur, they may cause the formation of a risk chain. Therefore, in the early stages of a PPP project, attention should be paid to the government's decision-making and the planning and design of the PPP project. In the life cycle of PPP projects, there should be focus on government regulation, delinquency of private, environmental pollution and public opposition.

An empirical study in 2011 showed that the three most important risk factors for Chinese PPP projects are government intervention, government corruption, and poor public decision-making processes [89], which is consistent with our research. In addition, we also need to make a special description of R19 (environmental risk), as China imposed an environmental tax in January 2011 [90]. With increasingly strict environmental protection policies, the price of raw materials in the construction industry has been generally rising, which leads to the rising cost of many construction projects which, finally, leads to the failure of projects. From the above analysis, we can see that the fundamental risks affecting PPP projects are not invariable, but rather, are closely related to the actual situation.

(2) From Figures 2 and 3, we found that the R7 (contract risk), R9 (financing risk), R12 (construction risk), R13 (project income risk), and R15 (operation and maintenance risk) have higher dependency values (R15 is in the middle of Figure 2, but in the Quadrant IV of Figure 3), indicating that they are easily affected by other risks. The occurrence of these risks is often caused by the accumulation of other risks and, when these risks occur, they can easily and directly cause the failure of the project, in agreement with the study of $\mathrm{Li}$ and Wang [18]. We can prevent such risk factors by controlling the occurrence of the other risks that have an impact on them. In addition, it should be realized that, as these risk factors are easily influenced by other risk factors, their own performance can reflect whether the risks affecting them have been well-controlled or not; that is to say, they can also serve as an indicator of the effect of risk management.

(3) In addition, it can be seen, from Figure 3, that no risk factors are located in Quadrant III, which reflects that there is no PPP risk that can be affected by many factors and can affect many factors, which is consistent with the research of Iyer et al. [19]. This is demonstrates that the key risk factors usually do not lead to project failure through a singular role, but rather, increase the impact on the project through a correlation between risks, cumulatively contributing to the failure of a PPP project.

\subsection{Comparison with Other Studies}

By comparing Iyer's study on the Indian highway PPP [19], Han's study on the Chinese brownfield remediation projects PPP [79], and Li's ISM study on Chinese PPP [18], we find that:

1. In Iyer's study on PPP of Indian highways [19], environmental risk was also at the bottom of the risk level, which could easily induce permit risk and direct political risk, eventually triggering schedule risk and cost overrun risk, which is consistent with our study and indicates that there are similarities between India and China, in this respect.

2. Comparing the PPP risk studies of Han [83] and Li [18], based on Chinese national conditions, Han believed the bottom of the risk was an immature legal system, inadequate experience, inadequate data and research, lack of a standard PPP contract template, private inability, and technique risk. Meanwhile, in Li's research, legal and policy risk, organization and coordination risk, rights, responsibility, and risk allocation between the co-operative parties were considered to be at the bottom. Although they each had different opinions of risk, their research was consistent: 
a government with weak supervision and ability, a private party with inability and irregularities, and the lack of organization and coordination between them, are projected at the bottom of the risk. These risks can be controlled, and the managers and decision-makers must work to control these risks.

3. Previous studies and this study, have found that both contract risk and financing risk can induce the failure of PPP projects, as triggered by construction risk (including schedule risk and cost overrun risk).

4. As for the MICMAC analysis, each study differed. In our study, there was no risk found in Quadrant III. In the study of Iyer [19], there was no risk in Quadrant III and IV; in the study of Li [14], there was no risk in Quadrant I; meanwhile, in the study of Han [83], there was risk in all quadrants. Therefore, in the present study and that of Iyer, the risks were more independent of each other. In contrast, the degree of risk interrelation in the studies of of Han and Li was higher. This difference was caused by one the of factors influencing the existence of ambiguity, which further proves the advantages of FISM-MICMAC method. For a different cutting coefficient, we could control the threshold value of correlation degree between various factors.

\subsection{Suggestions to the Government}

1. China's current PPP related policies and regulations are not perfect [91,92]. The government should summarize the many problems existing in current PPP projects, and formulate special PPP laws and regulations, as soon as possible, to protect the rights and interests of both parties, reduce the negative effects caused by changes in policies and regulations, and enhance the investment confidence of the private parties involved.

2. The choice of private sector is very important. A private sector with strong financing, technical ability, and high reputation will provide higher advantages in a PPP project, such as by realizing project appreciation, reducing financing risk, construction risk, operation and maintenance risk, and the risk of the project company violating laws and regulations. In No. 27 case in Appendix A, the wrong choice of private party leads to improper subcontracting of PPP projects, which ultimately leads to the failure of the PPP projects.

3. In addition, the government should regard PPP as a governance scheme, rather than a pragmatic economic tool [92]. Governmental departments should strengthen the PPP-relevant theoretical knowledge and practical experience, learn from similar projects, pay attention to the feasibility in early stages of the project, establish and perfect project decision-making mechanisms, and conduct in-depth research on the project for scientific planning and design. In addition, governmental departments should strengthen supervision and management, perfect their regulation systems, and reduce the PPP project risk that exists because of inadequate supervision.

4. The government should realize that the relationship with stakeholders in a PPP project is key to the sustainable development of the project $[93,94]$. The government should attach importance to the relationship with private parties, avoid the risk of governmental decision-making approval as much as possible, reduce late-project changes, avoid competition of similar projects caused by unreasonable commitments in the early stage, and gradually improve government credit.

5. In terms of PPP project contract, the rights and obligations of the public and private should be reasonably distributed, the contents (such as the distribution of benefits and risks) of dispute resolutions and renegotiation mechanisms should be clarified, and the behaviors of both parties should be regulated $[1,29,30]$. According to the actual situation of the project, a reasonable risk sharing mechanism should be determined for planning and design risks, financial risks, environmental risks, change risks, force majeure risk, and public opposition risks.

6. In the past, government departments have paid too much attention to economic evaluation while ignoring social evaluation when evaluating investment projects, leading to higher rates of public opposition [95]. In the implementation of PPP projects in the future, the period for soliciting public opinion should be extended, and public information should be kept open throughout the 
period for soliciting public opinion, so as to improve public participation, give full play to the role of public supervision, and ensure the normal operations of the project.

\subsection{Limitations of this Study}

This study, has some limitations:

1. The cases in this paper were consulted on the Internet, without field verification, meaning that certain risks may be missed. However, the combination method of case study, literature survey and expert interview makes up some deficiencies. Moreover, with the development of PPP projects, there will be increasingly similar projects. Future work will further supplement the cases.

2. Due to the limited number of PPP experts and their limited time availability, in addition to the means of on-site, we also used WeChat, e-mail and other methods in questionnaire survey, which may could have affected the quality of the questionnaire. It takes much time and effort to complete 380 triangular fuzzy numbers for the 20 risk correlations in one questionnaire. Some experts did not have enough patience and/or time to fill out the questionnaire, so the data through WeChat and e-mail are often chaotic. However, by taking the high impact of other risks on the R16 (force majeure risk) as an invalid judgment standard, the validity of the questionnaire could be guaranteed.

3. The risks in this study are mainly about PPP risks in China, and the relationships between analyzed risks are mainly applicable to the current situation in China. For example, the environmental risk (R19) is at the bottom of the risk hierarchy, which is related to China's sudden environmental policies. This may be not in line with other countries. However, the research methods and framework proposed in this study, can be applied to other studies on the relationship between PPP risks in different countries and regions.

4. A total of 20 items are identified as PPP project failure risk factors, which is a broadly representative result, suitable for the key risk factors identified in most PPP projects. However, in a specific project, depending on the specific situation of a PPP project, it may be appropriate to supplement or adjust the key risk factors. For example, in a PPP project for waste incineration, there is often public opposition, while in PPP projects for sponge cities or wetland parks, there is usually no public opposition. In these circumstances, public opposition risk (R17) should be adapted according to the characteristics of the PPP projects.

5. Although fuzzy theory reduces the subjectivity of expert judgment, it also increases the workload of data processing, requiring more resources to be invested into modeling and analysis. Thus, the method in this paper would benefit from programs to facilitate its application.

6. The triangular fuzzy number given by experts will have significant impact on the results. If there are disputes among experts about the risk relationship (the triangular fuzzy number of different experts varies greatly), it will be difficult to calculate the results. In addition, the selection of the intercept coefficient requires PPP experts with abundant experience and professional insight.

\section{Conclusions and Future Research}

There is a close interaction between PPP project risks, which has been neglected in relevant literature. Furthermore, the existing research on the interaction of PPP risks lacked consideration of complexity and ambiguity among risks. Based on this, this study has adopted the FISM-MICMAC method to discuss the risk factors and their hierarchical relationship of PPP projects. In addition, the method of FISM-MICMAC can provide a new risk assessment tool for risk management strategies in the field of construction management.

This study started by identifying key risk factors that lead to the failure of PPP projects, using a combination of various risk identification methods, such as case study, literature survey, and expert interviews, in order to identify 20 key risk factors that have an important impact on the success or failure of PPP projects. Then, FISM was used to discuss the PPP project risk factors and their 
hierarchical relationship, where the hierarchical structure chart of the key risk factors of PPP projects was constructed. Moreover, MICMAC was used to classify the driving power and dependence power of the risk factors, so as to solve the complex problems of the interaction and feedback relationships between key risk factors in PPP projects. The final sequence of factors, which have the obvious characteristics of network, was obtained as a result of an analysis of the dependencies between risk factors.

On the basis of this study, further research can be carried out in the future: (1) Although we have ascertained relationships of influence between risks, the degree of influence, impact speed, and impact mechanisms remain to be further studied; (2) The coupling effect between risk factors needs to be further analyzed, and the overall risk of the project under the coupling effect needs to be evaluated; (3) In future studies, risk factors for the failure of PPP projects can be combined with liability sharing to establish a liability sharing model under the interaction of risks, which can provide a decision-making basis for clearly defining responsibility and compensation for failure.

Author Contributions: Conceptualization, X.J.; methodology, X.J. and K.L.; software, K.L.; formal analysis, X.J. and B.X.; investigation, Y.L. and C.C.; data collection, K.L. and Y.L.; writing-original draft preparation, X.J. and K.L.; writing-review and editing, B.X.; funding acquisition, X.J. and Y.L.; supervision, X.J.

Funding: The research was supported by the National Natural Science Foundation of China (71672180), the Soft Science Research Plan of Department of Housing and Urban-Rural Development of Anhui Province in China (No. JS2016AHST0011), Innovation Research Plan of Anhui Construction Engineering Group in China (No. W2018JSZX0002).

Conflicts of Interest: There is no conflict of interest. 


\section{Appendix A Case Description and Risk Factor Extraction for 28 Failed PPP Projects}

Table A1. Project overview, problem description, and risk extraction of 28 failed PPP projects.

\begin{tabular}{|c|c|c|c|c|}
\hline No. & Project & Project Overview and Results & Failure or Problem Description & Risk Factor Extraction \\
\hline (1) & $\begin{array}{l}\text { Changchun Huilu } \\
\text { sewage treatment } \\
\text { plant }\end{array}$ & $\begin{array}{l}\text { Signed the contract in } 2000 \text { with an } \\
\text { investment scale of } \$ 32 \text { million dollars, } \\
\text { and defaulted on the water bill in } 2002 \text {. } \\
\text { Government made buyback in } 2005 .\end{array}$ & $\begin{array}{l}\text { The government abolished franchise schemes and } \\
\text { stopped paying water bills. It was difficult to maintain } \\
\text { the normal operations of the project. The sewage plant } \\
\text { has failed in the appeal. The project experienced two } \\
\text { years of legal disputes. }\end{array}$ & $\begin{array}{l}\text { Policy and regulatory change risk; Government } \\
\text { credit risk; Project income risk; Government } \\
\text { regulatory risk; Project company violates laws and } \\
\text { regulations; Environment risk. }\end{array}$ \\
\hline (2) & $\begin{array}{l}\text { Qingdao Veolia } \\
\text { sewage treatment } \\
\text { plant }\end{array}$ & $\begin{array}{l}\text { Signed in 2003, with an investment scale } \\
\text { of } \$ 42.8 \text { million. After renegotiation, } \\
\$ 32.7 \text { million of reconstruction in } 2015 \text {. }\end{array}$ & $\begin{array}{l}\text { The government had limited understanding of PPP. After } \\
\text { the contract was signed, the government thought the } \\
\text { price was unfair and unilaterally asked for renegotiation. } \\
\text { Lack of effective supervision and pollution incidents led } \\
\text { to public protests. }\end{array}$ & $\begin{array}{l}\text { Government credit risk; Lack of PPP co-operation } \\
\text { experience; Operation and maintenance risk; } \\
\text { Government regulatory risk; Project company } \\
\text { violates laws and regulations; Environmental risks; } \\
\text { Imperfect price adjustment mechanism; Public } \\
\text { opposition risk. }\end{array}$ \\
\hline (3) & $\begin{array}{l}\text { Wuhan Townsend } \\
\text { sewage treatment } \\
\text { plant }\end{array}$ & $\begin{array}{l}\text { Construction in 2001; } \$ 12.8 \text { million } \\
\text { investment; Government made buyback } \\
\text { in } 2004 .\end{array}$ & $\begin{array}{l}\text { The surrounding sewage network was not equipped, } \\
\text { there was no water source for operation, and the problem } \\
\text { of a sewage treatment fee was not solved. The project is } \\
\text { idle after completion. } \\
\text { Long-term failure to sign a contract after winning the bid. }\end{array}$ & $\begin{array}{l}\text { Government decision-making approval risks; } \\
\text { Changes in market demand; Imperfect price } \\
\text { adjustment mechanism; Facility matching risk. }\end{array}$ \\
\hline (4) & $\begin{array}{l}\text { Beijing No.10 water } \\
\text { plant }\end{array}$ & $\begin{array}{l}\text { The project was initiated in } 1998 \text { with an } \\
\text { investment scale of } \$ 326.6 \text { million and } \\
\text { started in 2012. It was not officially } \\
\text { operational in } 2015 \text {. }\end{array}$ & $\begin{array}{l}\text { Market supply and demand upside down, the risk was } \\
\text { mainly borne by investors. Banks did not lend. } \\
\text { Difficulties in land allocation and rising construction } \\
\text { costs. Network changes. Water prices raised public } \\
\text { opposition. }\end{array}$ & $\begin{array}{l}\text { Lengthy government decision-making and approval; } \\
\text { Planning and design risk; Changes in market } \\
\text { demand; Construction cost overrun; Public } \\
\text { opposition risk; Financing risk. }\end{array}$ \\
\hline (5) & $\begin{array}{l}\text { Shanghai Dayang } \\
\text { water plant }\end{array}$ & $\begin{array}{l}\text { Signed in } 1996 \text { with an investment of } \\
\$ 70 \text { million. Government made buyback } \\
\text { in } 2004 \text {. }\end{array}$ & $\begin{array}{l}\text { Delay in project approval. Water price below cost was } \\
\text { difficult to increase. Government fixed rate of return, } \\
\text { conflict with policy, renegotiation failed. }\end{array}$ & $\begin{array}{l}\text { Lengthy government decision-making and approval; } \\
\text { Imperfect price adjustment mechanism; } \\
\text { Unreasonable profit distribution; Government credit } \\
\text { risk; Policy and regulatory change risk. }\end{array}$ \\
\hline (6) & $\begin{array}{l}\text { Lianjiang zhongfa } \\
\text { water plant }\end{array}$ & $\begin{array}{l}\text { Signed in } 1997 \text {, invested } \$ 16.69 \text { million. } \\
\text { It sat idle and sued in } 1999 \text {, and } \\
\text { government made buyback in } 2009 .\end{array}$ & $\begin{array}{l}\text { Water quantity and price agreement could not be } \\
\text { implemented. The price of water was too high for the } \\
\text { public to afford, and the government could not continue } \\
\text { to pay for it. Chinese and French water companies } \\
\text { suffered losses. }\end{array}$ & $\begin{array}{l}\text { Lack of PPP co-operation experience; Government } \\
\text { official corruption; Government credit risk; Imperfect } \\
\text { price adjustment mechanism; Project income risk. }\end{array}$ \\
\hline (7) & $\begin{array}{l}\text { Shenyang No.9 } \\
\text { water plant }\end{array}$ & $\begin{array}{l}\text { Equity transfer negotiated in 1996, and } \\
\text { contract changed in } 2000 \text {. Government } \\
\text { made two buybacks in } 2001 \text { and in } 2006 .\end{array}$ & $\begin{array}{l}\text { The sewage treatment fee was higher than the market } \\
\text { price, and Shenyang water supply company lost money. } \\
\text { Contract negotiation and modification. }\end{array}$ & $\begin{array}{l}\text { Lack of PPP co-operation experience; Government } \\
\text { official corruption; Imperfect price adjustment } \\
\text { mechanism; Unreasonable profit distribution. }\end{array}$ \\
\hline
\end{tabular}


Table A1. Cont.

\begin{tabular}{|c|c|c|c|c|}
\hline No. & Project & Project Overview and Results & Failure or Problem Description & Risk Factor Extraction \\
\hline (8) & $\begin{array}{l}\text { Chengdu Veolia } \\
\text { No.6 water plant }\end{array}$ & $\begin{array}{l}\text { Built in } 2000 \text { with an investment of } \\
\$ 106.5 \text { million. It was put into operation } \\
\text { in February 2002. The project suffered } \\
\text { heavy losses. }\end{array}$ & $\begin{array}{l}\text { Some of the projects changed for legal reasons were BT } \\
\text { projects. The economic situation was not reasonably } \\
\text { predicted and the water supply market was seriously } \\
\text { surplus. The water company suffered heavy losses. }\end{array}$ & $\begin{array}{l}\text { Planning and design risk; Lack of PPP co-operation } \\
\text { experience; Unreasonable profit distribution; } \\
\text { Changes in market demand; Policy and regulatory } \\
\text { change risk. }\end{array}$ \\
\hline (9) & $\begin{array}{l}\text { Quanzhou } \\
\text { Donghai municipal } \\
\text { sewage treatment } \\
\text { plant }\end{array}$ & $\begin{array}{l}\text { In Phase I, investment was } 6.66 \text { million } \\
\text { yuan, bidding price was } \$ 0.12 \text { dollar/m3. } \\
\text { Project completed in } 2011 \text { and did not } \\
\text { implement VFM. }\end{array}$ & $\begin{array}{l}\text { During the construction period, the government required } \\
\text { speeding up of the construction, with construction cost } \\
\text { overruns. According to the contract, the adjustment } \\
\text { period of water price was five years, which led to great } \\
\text { market risks. Suspension of franchise period and } \\
\text { adjustment ambiguity were controversial. }\end{array}$ & $\begin{array}{l}\text { Government intervention; Construction cost overrun; } \\
\text { Imperfect price adjustment mechanism; Imperfect } \\
\text { dispute settlement mechanism. }\end{array}$ \\
\hline (11) & $\begin{array}{l}\text { Shanghai } \\
\text { Huqingping } \\
\text { (Huyu) expressway }\end{array}$ & $\begin{array}{l}\text { Won the bid in 2000, completed the } \\
\text { whole line in 2005. In July 2008, the } \\
\text { contract was suspended ahead of } \\
\text { schedule and government withdrew the } \\
\text { operation. }\end{array}$ & $\begin{array}{l}\text { The selection process of social capital was not } \\
\text { transparent, the project company had poor management, } \\
\text { and there were many violations. The project capital and } \\
\text { financial supervision were not in place, the project } \\
\text { payment is in arrears, and the actual income is low. } \\
\text { Sewage discharge was not up to standard, and the }\end{array}$ & $\begin{array}{l}\text { Government regulatory risk; Construction risk; } \\
\text { Operation and maintenance risks; Bidding risks; } \\
\text { Changes in market demand; Project company } \\
\text { violates laws and regulations. }\end{array}$ \\
\hline (13) & $\begin{array}{l}\text { Hangzhou Bay } \\
\text { sea-crossing bridge }\end{array}$ & $\begin{array}{l}\text { Started in } 2003 \text { and opened in } 2008 \text { with } \\
\text { a total investment of about } \$ 1675.5 \\
\text { million yuan. It lost money in } 2013 \text { and } \\
\text { government made buyback. }\end{array}$ & $\begin{array}{l}\text { Private capital transfer and withdrawal, the government } \\
\text { to buy back } 80 \% \text { of the equity. During the construction } \\
\text { period, the government repeatedly proposed to change } \\
\text { the rate of return on investment. Suffer from interest rate } \\
\text { increase inflation, serious losses, unable to recover the } \\
\text { principal on schedule. }\end{array}$ & $\begin{array}{l}\text { Planning and design risk; Construction cost overrun; } \\
\text { Change in market demand; Parallel project } \\
\text { competitive risk; Contract risk; Economic risk. }\end{array}$ \\
\hline (14) & $\begin{array}{l}\text { Quanzhou } \\
\text { Wiliongtong bridge }\end{array}$ & $\begin{array}{l}\text { Project started in May } 1995 \text { with a total } \\
\text { investment of } \$ 35.5 \text { million and was put } \\
\text { into operation in } 1997 \text {. Government } \\
\text { made buyback in } 2016 \text {. }\end{array}$ & $\begin{array}{l}\text { Quanzhou government to build a number of free } \\
\text { competitive Bridges to disperse traffic. Defects of legal } \\
\text { contract, unclear division of responsibilities and rights. } \\
\text { The contract stipulated that the concession period exceed } \\
\text { the state regulation, and fee increase was not permitted }\end{array}$ & $\begin{array}{l}\text { Policy and regulatory change risk; Parallel project } \\
\text { competitive risk; Government credit risk; Insufficient } \\
\text { expense payment; Incomplete contract risk. }\end{array}$ \\
\hline
\end{tabular}


Table A1. Cont.

\begin{tabular}{|c|c|c|c|c|}
\hline No. & Project & Project Overview and Results & Failure or Problem Description & Risk Factor Extraction \\
\hline (15) & $\begin{array}{l}\text { Changtan west } \\
\text { expressway }\end{array}$ & $\begin{array}{l}\text { Started construction in } 2004 \text { with an } \\
\text { investment of } \$ 122.1 \text { million and opened } \\
\text { to traffic in } 2007 \text {. The project suffered } \\
\text { heavy losses. }\end{array}$ & $\begin{array}{l}\text { The government defaulted, the compensation for land } \\
\text { expropriation and demolition failed, and the toll station } \\
\text { was repeatedly changed during construction. A number } \\
\text { of competitive toll highways existed. }\end{array}$ & $\begin{array}{l}\text { Government intervention; Parallel project } \\
\text { competitive risk; Insufficient expense payment; } \\
\text { Planning and design risk; Completion risk. }\end{array}$ \\
\hline (17) & $\begin{array}{l}\text { Xianyang Weihe } \\
\text { river No.3 bridge }\end{array}$ & $\begin{array}{l}\text { Started in } 2003 \text { and was opened to traffic } \\
\text { in } 2004 \text {, with a total investment of about } \\
\$ 17.0 \text { million. Government made } \\
\text { buyback in } 2011 .\end{array}$ & $\begin{array}{l}\text { Affected by SARS in the early stages of project } \\
\text { construction, labor shortage. The traffic volume forecast } \\
\text { was insufficient, the construction scale was insufficient. } \\
\text { In the operation stage, the service quality was not up to } \\
\text { standard, and traffic jams on the bridge caused public } \\
\text { opposition. }\end{array}$ & $\begin{array}{l}\text { Government credit risk; Force majeure risk; Parallel } \\
\text { project competitive risk; Public opposition risk; } \\
\text { Planning and design risk; Organizational } \\
\text { coordination risk. }\end{array}$ \\
\hline (18) & $\begin{array}{l}\text { Shandong } \\
\text { Zhonghua power } \\
\text { generation project }\end{array}$ & $\begin{array}{l}\text { Initiated in } 1997 \text { and completed in } 2004 . \\
\text { Project collaboration is in trouble. }\end{array}$ & $\begin{array}{l}\text { Reform of electric power system in the operation period. } \\
\text { The government promised that the minimum purchase } \\
\text { power was insufficient, so could not co-operate. In 2002, } \\
\text { fees were reduced and revenues reduced. }\end{array}$ & $\begin{array}{l}\text { Lack of PPP co-operation experience; Government } \\
\text { credit risk; Changes in market demand; Contract } \\
\text { risk; Policy and regulatory change risk. }\end{array}$ \\
\hline (19) & $\begin{array}{l}\text { Nanjing Yangtze } \\
\text { river three bridges }\end{array}$ & $\begin{array}{l}\text { Started construction in } 2002 \text { and opened } \\
\text { to traffic in } 2005 \text { with a total investment } \\
\text { of } \$ 438.7 \text { million. After opening to } \\
\text { traffic, the project suffered serious losses. }\end{array}$ & $\begin{array}{l}\text { The location was remote, the road network on both sides } \\
\text { was not perfect, the traffic flow was seriously lower than } \\
\text { expected, and the financial cost was high, resulting in a } \\
\text { project loss. In 2009, the government negotiated an } \\
\text { extension of the franchise to } 30 \text { years. }\end{array}$ & $\begin{array}{l}\text { Planning and design risk; project income risk; } \\
\text { Facility matching risk. }\end{array}$ \\
\hline (20) & $\begin{array}{l}\text { Tianjin } \\
\text { Shuanggang waste } \\
\text { incineration power } \\
\text { plant }\end{array}$ & $\begin{array}{l}\text { Started construction in } 2003 \text { and went } \\
\text { into operation in } 2005 \text { with a total } \\
\text { investment of } \$ 76.7 \text { million. Project is in } \\
\text { trouble. }\end{array}$ & $\begin{array}{l}\text { Waste incineration pollution, poor government } \\
\text { supervision, public opposition. The amount of subsidy } \\
\text { promised by the government was not clear, and the } \\
\text { project was not profitable enough } \\
\text { The technology was not mature and the argument was }\end{array}$ & $\begin{array}{l}\text { Planning and design risk; Environmental risk; } \\
\text { Government credit risk; insufficient expense } \\
\text { payment; Government regulatory risk. }\end{array}$ \\
\hline (21) & $\begin{array}{l}\text { Shandong Heze } \\
\text { waste-to-power } \\
\text { plant }\end{array}$ & $\begin{array}{l}\text { In 2013, a co-operation agreement was } \\
\text { signed, with an initial investment of } \\
\$ 49.7 \text { million. The project suffered } \\
\text { serious losses. }\end{array}$ & $\begin{array}{l}\text { not sufficient. Accumulated losses of more than } \$ 2.1 \\
\text { million in four years of operation. Failure to implement } \\
\text { government subsidy schemes. Mix-in coal amount } \\
\text { exceeded national regulation, became small thermal } \\
\text { power plant. }\end{array}$ & $\begin{array}{l}\text { Planning and design risk; Policy and regulatory } \\
\text { change risks; Government credit risk; Project } \\
\text { changes risk; Project income risk; Project company } \\
\text { violates laws and regulations. }\end{array}$ \\
\hline (22) & $\begin{array}{l}\text { Beijing Liulitun } \\
\text { waste-to-power } \\
\text { plant }\end{array}$ & $\begin{array}{l}\text { Approved in } 2005 \text { and planned to start } \\
\text { construction in } 2007 \text {. In 2007, the state } \\
\text { environmental protection } \\
\text { administration ordered a moratorium, } \\
\text { and the project was halted in } 2011 .\end{array}$ & $\begin{array}{l}\text { Project planning was lagging behind, the site was close to } \\
\text { a diversion canal and residential air outlet, and the odor } \\
\text { affected the surrounding environment, strongly opposed } \\
\text { by residents. The state environmental protection } \\
\text { administration ordered a moratorium on construction, } \\
\text { and the project has been suspended for a long time. }\end{array}$ & $\begin{array}{l}\text { Government decision-making approval risk; } \\
\text { Planning and design risk; Public opposition risk; } \\
\text { Environmental risk. }\end{array}$ \\
\hline
\end{tabular}


Table A1. Cont.

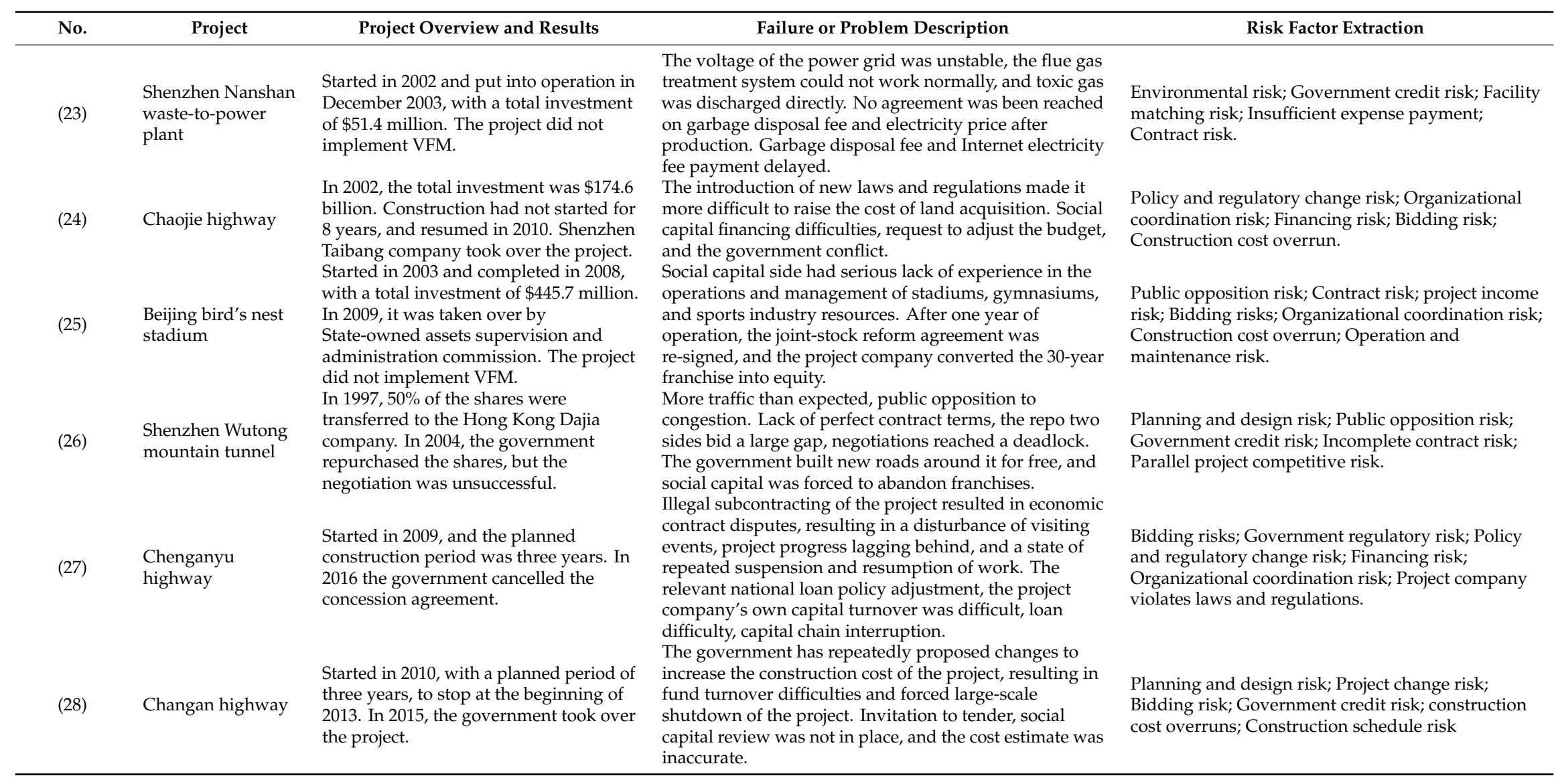


Table A2. 28 failed projects and their links to websites.

\begin{tabular}{|c|c|c|}
\hline No. & Project & Links to Websites (Accessed on 19 September 2019) \\
\hline$(1)$ & Changchun Huilu sewage treatment plant & http://huanbao.bjx.com.cn/news/20150305/594592.shtml \\
\hline$(2)$ & Qingdao Veolia sewage treatment plant & http://www.h2o-china.com/news/243917.html \\
\hline (3) & Wuhan Townsend sewage treatment plant & http://www.h2o-china.com/news/243917.html \\
\hline$(4)$ & Beijing No.10 water plant & http://www.xcar.com.cn/bbs/viewthread.php?tid=26304486 \\
\hline (5) & Shanghai Dayang water plant & http://blog.sina.com.cn/s/blog_99ca571e0102vzcd.html \\
\hline$(6)$ & Lianjiang zhongfa water plant & http://mini.eastday.com/mobile/180326172138091.html\# \\
\hline (7) & Shenyang No.9 water plant & http://mini.eastday.com/mobile/180326172138091.html\# \\
\hline$(8)$ & Chengdu Veolia No.6 water plant & http://www.cspea.org.cn/article/hydt/hydt/201708/20170800006038.shtml \\
\hline (9) & Quanzhou Donghai municipal sewage treatment plant & https://max.book118.com/html/2017/1126/141657699.shtm \\
\hline (10) & Lanzhou Veolia water & http://www.tanpaifang.com/ppp/201612/0757836_4.html \\
\hline (11) & Shanghai Huqingping (Huyu) expressway & https://www.zjtcn.com/baike/hygsgl \\
\hline (12) & Jinzhou municipal sewage treatment plant & https://www.tianyancha.com/company/2333146105 \\
\hline (13) & Hangzhou Bay Sea-Crossing Bridge & http://www.360doc.com/content/17/0210/11/366082_627991592.shtml \\
\hline$(14)$ & Quanzhou Wiliongtong bridge & http://opinion.caixin.com/2014-09-25/100732936.html \\
\hline (15) & Changtan west expressway & https://www.sohu.com/a/217236864_99929980 \\
\hline (16) & Wuhan Yangtze river three Bridges & https://www.sohu.com/a/205755812_100012673 \\
\hline (17) & Xianyang Weihe river No.3 bridge & https://www.docin.com/p-1434785098.html \\
\hline (18) & Shandong Zhonghua power generation project & https://www.zhihu.com/question/28741657?sort=created \\
\hline (19) & Nanjing Yangtze river three Bridges & http://www.360doc.com/content/16/0722/13/366082_577533747.shtml \\
\hline (20) & Tianjin Shuanggang waste incineration power plant & http://mini.eastday.com/mobile/180326172138091.html\# \\
\hline$(21)$ & Shandong Heze waste-to-power plant & http://huanbao.bjx.com.cn/news/20171227/870276.shtml \\
\hline$(22)$ & Beijing Liulitun waste-to-power plant & https://news.qq.com/a/20110120/000136.htm \\
\hline (23) & Shenzhen Nanshan waste-to-power plant & http://www.sohu.com/a/214527318_100053329 \\
\hline (24) & Chaojie highway & http://www.gongdaogc.com/show.asp?id=40917 \\
\hline (25) & Beijing bird's nest stadium & https://www.sohu.com/a/142496023_798724 \\
\hline (26) & Shenzhen Wutong mountain tunnel & https://www.sohu.com/a/133919852_739751 \\
\hline (27) & Chenganyu highway & http://www.sohu.com/a/19689655_116913 \\
\hline (28) & Changan highway & http://bbs.tianya.cn/post-293-152478-1.shtml \\
\hline
\end{tabular}




\section{Appendix B Questionnaire on the Risk Relationship of PPP Projects}

Dear experts,

Hello! Research Group of Dr. Jiang, School of Civil Engineering, Hefei University of Technology, is carrying out research on " the risk relationship of PPP projects ". Our research group sincerely invites you to give your opinion on this survey based on your past experience and relevant knowledge in PPP projects. Your opinion is very valuable and will play an important role in our research. The data and information collected in this questionnaire will only be used for academic research and will not negatively affect your daily work and life. Thank you for your understanding and support!

Jiang Group

Table 1 shows the list of key risk factors of PPP and their meaning explanation. This survey gives a list of risk factors according to the past practice in the 28 failure PPP project cases, related literature survey, and expert interview. After familiarizing the risk factors in Table 1, please complete Table 3 according to the reminders in Table 2.

Table A3. Risk factors and their meaning explanation for PPP projects.

\begin{tabular}{|c|c|}
\hline Risk Factor & Meaning Explanation \\
\hline $\begin{array}{l}\text { Policy and regulatory } \\
\text { change risk (R2) }\end{array}$ & $\begin{array}{l}\text { Existing PPP-related policies and regulations are unsound, including low legislative level, poor } \\
\text { operability, and conflict. } \\
\text { 现有的PPP相关政策法规不健全、立法层级低、可操作性差、相互冲突; }\end{array}$ \\
\hline $\begin{array}{l}\text { Government } \\
\text { regulatory risk (R4) }\end{array}$ & $\begin{array}{l}\text { The government does not have adequate supervision over the project, and the supervision } \\
\text { system is not perfect. } \\
\text { 政府对项目监管不到位, 监管体系不完善。 }\end{array}$ \\
\hline Bidding risk (R6) & $\begin{array}{l}\text { These are vicious competition or insufficient competition among private capital, unreasonable } \\
\text { bidding, tendering procedures, lax qualification examination conditions and insufficient } \\
\text { supervision, etc. The government choose the incompetent private capital. } \\
\text { 社会资本方之间存在恶性竞争或竞争不足、招标投标程序不合理、资格审查条件不严格、监督 } \\
\text { 力度不够等。政府选择了不称职的私人资本方。 }\end{array}$ \\
\hline Contract risk (R7) & $\begin{array}{l}\text { There are problems with the contract design, including: contract ambiguity, contract document } \\
\text { conflict, contract storage not in place, imperfect price adjustment mechanism, unreasonable } \\
\text { profit distribution, imperfect dispute settlement mechanism, and incomplete contract. Risk } \\
\text { allocation and income distribution are not reasonable. The scope of rights and responsibilities is } \\
\text { not clear. } \\
\text { 合同设计存在问题，包括:合同歧义、合同文件冲突、合同保管不到位、价格调节机制不完善、 } \\
\text { 利润分配不合理、纠纷解决机制不完善、合同不完整等。风险配置和收益分配不合理。权利和 } \\
\text { 责任范围不明确。 }\end{array}$ \\
\hline Financing risk (R9) & $\begin{array}{l}\text { The financial market is not sound, financing channels are not smooth, financing structure is not } \\
\text { reasonable with high debt ratio. It is difficult to raise funds for the project. } \\
\text { 融资市场不健全，融资渠道不畅通，融资结构不合理，负债率高。为这个项目筹集资金 } \\
\text { 是困难的 }\end{array}$ \\
\hline
\end{tabular}


Table A3. Cont.

\begin{tabular}{|c|c|}
\hline Risk Factor & Meaning Explanation \\
\hline Economic risk (R10) & $\begin{array}{l}\text { It refers to the uncertainty of expected cash flow of PPP projects caused by changes in } \\
\text { macroeconomic factors and fragility of the financial system, as well as the possibility of expected } \\
\text { income loss of PPP projects caused by changes in exchange rate, interest rate, commodity price } \\
\text { and inflation. } \\
\text { 指宏观经济因素的变动以及金融体系内在的不稳定性和脆弱性而带来的PPP项目预期现金流的 } \\
\text { 不确定性, 以及由于汇率、利率和商品价格变动以及通货膨胀带来的PPP项目预期收益损失的 } \\
\text { 可能性变动 }\end{array}$ \\
\hline $\begin{array}{l}\text { Project change risk } \\
\text { (R11) }\end{array}$ & $\begin{array}{l}\text { The project changes, including the change of the construction object, the change of the operation } \\
\text { scope, the change of the contract } \\
\text { 项目变更, 包括建设对象的变更、运营范围的变更、合同的变更 }\end{array}$ \\
\hline $\begin{array}{l}\text { Construction risk } \\
\text { (R12) }\end{array}$ & $\begin{array}{l}\text { Project construction management level is poor. Construction cost overruns. Project delays. The } \\
\text { quality is not up to standard. Project construction cannot be completed. } \\
\text { 项目建设管理水平较差。建设成本超支。项目延迟。质量不符合标准。项目建设不能完工。 }\end{array}$ \\
\hline $\begin{array}{l}\text { Project income risk } \\
\text { (R13) }\end{array}$ & $\begin{array}{l}\text { Due to the change of market demand or insufficient expense payment, project investors cannot } \\
\text { recover the investment cost or achieve the predetermined income level } \\
\text { 市场需要的变化或者支付能力不足, 导致项目投资者不能收回投资成本或不能达到预定收益水 } \\
\text { 平 }\end{array}$ \\
\hline $\begin{array}{l}\text { Parallel project } \\
\text { competitive risk (R14) }\end{array}$ & $\begin{array}{l}\text { The government or other investors build or rebuild similar projects, which form substantial } \\
\text { commercial competition with the original PPP projects. } \\
\text { 政府或其他投资人新建或改建相似的项目，与原有项目形成实质性商业竞争 }\end{array}$ \\
\hline $\begin{array}{l}\text { Operation and } \\
\text { maintenance risk (R15) }\end{array}$ & $\begin{array}{l}\text { The project company's operation efficiency is low. The price of raw materials, fuels and power } \\
\text { increases. The equipment is of poor quality and often maintained. The operation cost and } \\
\text { maintenance cost increase. } \\
\text { 项目公司运营效率低。原材料、燃料和电力价格上涨。设备质量差, 经常维修。运营和维护成 } \\
\text { 本增加。 }\end{array}$ \\
\hline $\begin{array}{l}\text { Force majeure risk } \\
\text { (R16) }\end{array}$ & $\begin{array}{l}\text { Force majeure means that both parties have no control over the event or situation that cannot be } \\
\text { avoided or overcome before the signing of the contract, such as war, embargo, terrorism, and } \\
\text { other social force majeure; floods, typhoons, earthquakes, fires, epidemics, and other natural } \\
\text { disasters. } \\
\text { 不可抗力是指双方合同签订前无法避免或克服的事件或情况, 如战争、禁运、恐怖主义等社会 } \\
\text { 不可抗力;洪水、台风、地震、火灾、疫病等自然灾害 }\end{array}$ \\
\hline $\begin{array}{l}\text { Public opposition risk } \\
\text { (R17) }\end{array}$ & $\begin{array}{l}\text { Due to the lack of protection of public interests, the public is dissatisfied with the project and } \\
\text { even opposes it. In order to protect the public interest and social stability, the government } \\
\text { usually negotiates with private capital. } \\
\text { 由于社会公共利益得不到保护或受损, 引起公众对项目产生不满甚至反对项目建设, 而政府为 } \\
\text { 维护公众利益和社会稳定, 通常会与私人资本谈判。 }\end{array}$ \\
\hline $\begin{array}{l}\text { Organizational } \\
\text { coordination risk (R18) }\end{array}$ & $\begin{array}{l}\text { Coordination between government departments is difficult. The organization and coordination } \\
\text { ability of the project company is insufficient. Communication costs among participants are } \\
\text { increase, and conflicts arise among participants. } \\
\text { 政府部门之间协调困难。项目公司组织协调能力不足。参与者之间的沟通成本增加, 参与者之 } \\
\text { 间存在冲突。 }\end{array}$ \\
\hline $\begin{array}{l}\text { Environmental risk } \\
\text { (R19) }\end{array}$ & $\begin{array}{l}\text { During the implementation of the project, the improvement of environmental protection } \\
\text { standards may lead to the increase of investment in new equipment. The project company failed } \\
\text { to take effective control measures and emergency measures due to the environmental damage. } \\
\text { 在项目实施过程中, 环保标准的提高可能会导致新设备投资的增加。项目公司未能采取有效的 } \\
\text { 控制措施和应急措施导致环境破坏。 }\end{array}$ \\
\hline $\begin{array}{l}\text { Project company } \\
\text { violates laws and } \\
\text { regulations (R20) }\end{array}$ & $\begin{array}{l}\text { During the implementation of the project, the project company or private capital violates laws } \\
\text { and regulations, such as using projects for fraud or modifying the project data maliciously。 } \\
\text { 在项目实施过程中, 项目公司或私人资本方违反法律法规, 比如, 利用项目进行欺计或者恶意 } \\
\text { 修改项目数据 }\end{array}$ \\
\hline
\end{tabular}

According to the scoring in Table 2, please judge the mutual relationship of two factors, and fill the evaluation results into the corresponding positions in Table 3. 
Table A4. Language operator, triangular fuzzy number, and scoring.

\begin{tabular}{ccc}
\hline Language Operator. & Triangular Fuzzy Number $\left(\tilde{d}_{i j}^{k}\right)$ & Scoring \\
\hline Very low impact (VL) & $(0,0,0.25)$ & 1 \\
\hline Low impact (L) & $(0,0.25,0.5)$ & 2 \\
\hline medium impact (M) & $(0.25,0.5,0.75)$ & 3 \\
\hline High impact (H) & $(0.5,0.75,1)$ & 4 \\
\hline Very high impact (VH) & $(0.75,1,1)$ & 5 \\
\hline
\end{tabular}

Example: when you consider the impact of the risk factor R1 on R2 to be very low impact, you should fill the " 1 " in the second row and third column of Table 3.

Table A5. Mutual relationship intensity between two risk factors.

\begin{tabular}{|c|c|c|c|c|c|c|c|c|c|c|c|c|c|c|c|c|c|c|c|c|}
\hline & R1 & R2 & R3 & R4 & R5 & R6 & R7 & R8 & R9 & R10 & R11 & R12 & R13 & R14 & R15 & R16 & R17 & R18 & R19 & R20 \\
\hline $\mathrm{R} 1$ & 0 & & & & & & & & & & & & & & & & & & & \\
\hline $\mathrm{R} 2$ & & 0 & & & & & & & & & & & & & & & & & & \\
\hline $\mathrm{R} 3$ & & & 0 & & & & & & & & & & & & & & & & & \\
\hline $\mathrm{R} 4$ & & & & 0 & & & & & & & & & & & & & & & & \\
\hline R5 & & & & & 0 & & & & & & & & & & & & & & & \\
\hline R6 & & & & & & 0 & & & & & & & & & & & & & & \\
\hline R7 & & & & & & & 0 & & & & & & & & & & & & & \\
\hline $\mathrm{R} 8$ & & & & & & & & 0 & & & & & & & & & & & & \\
\hline R9 & & & & & & & & & 0 & & & & & & & & & & & \\
\hline R10 & & & & & & & & & & 0 & & & & & & & & & & \\
\hline R11 & & & & & & & & & & & 0 & & & & & & & & & \\
\hline R12 & & & & & & & & & & & & 0 & & & & & & & & \\
\hline R13 & & & & & & & & & & & & & 0 & & & & & & & \\
\hline R14 & & & & & & & & & & & & & & 0 & & & & & & \\
\hline R15 & & & & & & & & & & & & & & & 0 & & & & & \\
\hline R16 & & & & & & & & & & & & & & & & 0 & & & & \\
\hline R17 & & & & & & & & & & & & & & & & & 0 & & & \\
\hline R18 & & & & & & & & & & & & & & & & & & 0 & & \\
\hline R19 & & & & & & & & & & & & & & & & & & & 0 & \\
\hline R20 & & & & & & & & & & & & & & & & & & & & 0 \\
\hline
\end{tabular}

Note: the risk factors affecting are column and the risk factors bearing the influence are row; there is an asymmetric relationship between risk factors. Please complete this questionnaire as soon as possible. Thank you again for your patience.

\section{References}

1. Ma, G.F.; Du, Q.J.; Wang, K.D. A Concession Period and Price Determination Model for PPP Projects: Based on Real Options and Risk Allocation. Sustainability 2018, 10, 21.

2. Hodge, G.A.; Greve, C. On public-private partnership performance: A contemporary review. Public Work. Manag. Policy 2017, 22, 55-78. [CrossRef]

3. Delorme, C.D.; Thompson, H.G.; Warren, R.S. Public infrastructure and private productivity: A stochastic-frontier approach. J. Macroecon. 1999, 21, 563-576. [CrossRef]

4. Yuan, J.; Wang, C.; Skibniewski, M.J.; Li, Q. Developing Key Performance Indicators for Public-Private Partnership Projects: Questionnaire Survey and Analysis. J. Manag. Eng. 2012, 28, 252-264. [CrossRef]

5. BANK, W. Private Participation in Infrastructure Database. Available online: http://ppi.worldbank.org/ customquery (accessed on 19 September 2019). 
6. Wegrzyn, J. The perception of critical success factors for PPP projects in different stakeholders groups. Entrep. Bus. Econ. Rev. 2016, 4, 81-92. [CrossRef]

7. Wojewnik-Filipkowska, A.; Trojanowski, D. Principles of public-private partnership financing-Polish experience. J. Prop. Invest. Financ. 2013, 31, 329-344. [CrossRef]

8. Tang, L.; Shen, Q.; Cheng, E.W.L. A review of studies on Public-Private Partnership projects in the construction industry. Int. J. Proj. Manag. 2010, 28, 683-694. [CrossRef]

9. Heravi, G.; Hajihosseini, Z. Risk Allocation in Public-Private Partnership Infrastructure Projects in Developing Countries: Case Study of the Tehran-Chalus Toll Road. J. Infrastruct. Syst. 2012, 18, 210-217. [CrossRef]

10. Osei-Kyei, R.; Chan, A.P.C. Review of studies on the critical success factors for public-private partnership (PPP) projects from 1990 to 2013. Int. J. Proj. Manag. 2015, 33, 1335-1346. [CrossRef]

11. Xu, Y.; Yeung, J.F.Y.; Chan, A.P.C.; Chan, Q.W.M.; Wang, S.Q.; Ke, Y. Developing a risk assessment model for PPP projects in China-A fuzzy synthetic evaluation approach. Autom. Constr. 2010, 19, 929-943. [CrossRef]

12. Shen, L.Y.; Platten, A.; Deng, X.P. Role of public private partnerships to manage risks in public sector projects in Hong Kong. Int. J. Proj. Manag. 2006, 24, 587-594. [CrossRef]

13. Chen, C.; Doloi, H. BOT application in China: Driving and impeding factors. Int. J. Proj. Manag. 2008, 26, 388-398. [CrossRef]

14. Ministry of Finance. Notice of Standardizing Project Library in PPP Integrated Information Platform "No. 92"; Ministry of Finance, Ed.; Ministry of Finance: Beijing, China, 2017.

15. Bridata. Data Custom Download. Available online: http://bridata.com/t\#/tools (accessed on 19 September 2019).

16. Liang, Y.; Wang, H. Sustainable Performance Measurements for Public-Private Partnership Projects: Empirical Evidence from China. Sustainability 2019, 11, 3653. [CrossRef]

17. Ke, Y.J.; Wang, S.Q.; Chan, A.P.C.; Lam, P.T.I. Preferred risk allocation in China's public-private partnership (PPP) projects. Int. J. Proj. Manag. 2010, 28, 482-492. [CrossRef]

18. Li, Y.; Wang, X.Y. Using fuzzy analytic network process and ISM methods for risk assessment of public-private partnership: A China perspective. J. Civ. Eng. Manag. 2019, 25, 168-183. [CrossRef]

19. Iyer, K.C.; Sagheer, M. Hierarchical Structuring of PPP Risks Using Interpretative Structural Modeling. J. Constr. Eng. Manag. 2010, 136, 151-159. [CrossRef]

20. Heinrich, H.W. Industrial Accident Prevention; McGraw-Hill: New York, NY, USA, 1979.

21. Bird, F.E. Management Guide to Loss Control; International Loss Control Institute: Atlanta, GA, USA, 1974.

22. Choi, J.H.; Chung, J.; Lee, D.J. Risk perception analysis: Participation in China's water PPP market. Int. J. Proj. Manag. 2010, 28, 580-592. [CrossRef]

23. Ke, Y.; Wang, S.; Chan, A.P.C. Risk Allocation in Public-Private Partnership Infrastructure Projects: Comparative Study. J. Infrastruct. Syst. 2010, 16, 343-351. [CrossRef]

24. Li, J.; Zou, P.X.W. Fuzzy AHP-based risk assessment methodology for PPP projects. J. Constr. Eng. Manag. 2011, 137, 1205-1209. [CrossRef]

25. Zhang, S.; Chan, A.P.C.; Feng, Y.; Duan, H.; Ke, Y. Critical review on PPP Research-A search from the Chinese and International Journals. Int. J. Proj. Manag. 2016, 34, 597-612. [CrossRef]

26. Liu, Y. Identification of Risk Factors Affecting PPP Waste-to-Energy Incineration Projects in China: A Multiple Case Study. Adv. Civ. Eng. 2018, 16, 4983523. [CrossRef]

27. Song, J.; Sun, C.; Xia, B.; Liu, S.; Skitmore, M. Risk identification for PPP waste-to-energy incineration projects in China. Energy Policy 2013, 61, 953-962. [CrossRef]

28. Jayasuriya, S. Challenges in public private partnerships in construction industry. Built Environ. Proj. Asset Manag. 2019, 9, 172-185. [CrossRef]

29. Shrestha, A.; Chan, T.K.; Aibinu, A.A.; Martek, L.; Chen, C.; Asce, A.M. Risk Allocation Inefficiencies in Chinese PPP Water Projects. J. Constr. Eng. Manag. 2018, 144, 04018013. [CrossRef]

30. Xu, Y.; Chan, A.P.C.; Yeung, J.F.Y. Developing a Fuzzy Risk Allocation Model for PPP Projects in China. J. Constr. Eng. Manag. 2010, 136, 894-903. [CrossRef]

31. Jin, X.H. Model for efficient risk allocation in privately financed public infrastructure projects using neuro-fuzzy techniques. J. Constr. Eng. Manag. 2011, 137, 1003-1014. [CrossRef]

32. Ameyaw, E.E.; Chan, A.P.C. Risk allocation in public-private partnership water supply projects in Ghana. Constr. Manag. Econ. 2015, 33, 187-208. [CrossRef]

33. Zou, P.X.W.; Li, J. Risk identification and assessment in subway projects: Case study of Nanjing Subway Line 2. Constr. Manag. Econ. 2010, 28, 1219-1238. [CrossRef] 
34. Wu, Y.; Xu, C.; Li, L.; Wang, Y.; Chen, K.; Xu, R. A risk assessment framework of PPP waste-to-energy incineration projects in China under 2-dimension linguistic environment. J. Clean. Prod. 2018, 183, 602-617. [CrossRef]

35. Mazher, K.M.; Chan, A.P.C.; Zahoor, H.; Khan, M.I. Fuzzy Integral-Based Risk-Assessment Approach for Public-Private Partnership Infrastructure Projects. J. Constr. Eng. Manag. 2018, 144, 04018111. [CrossRef]

36. Thomas, A.V.; Kalidindi, S.N.; Ganesh, L.S. Modelling and assessment of critical risks in BOT road projects. Constr. Manag. Econ. 2006, 24, 407-424. [CrossRef]

37. Bai, L.B.; Li, Y.; Du, Q.; Xu, Y. A Fuzzy Comprehensive Evaluation Model for Sustainability Risk Evaluation of PPP Projects. Sustainability 2017, 9, 1890. [CrossRef]

38. Zhang, L.; Sun, X.J.; Xue, H. Identifying critical risks in Sponge City PPP projects using DEMATEL method: A case study of China. J. Clean. Prod. 2019, 226, 949-958. [CrossRef]

39. Zheng, C.J.; Yuan, J.; Li, L.; Skibniewski, M.J. Process-Based Identification of Critical Factors for Residual Value Risk in China's Highway PPP Projects. Adv. Civ. Eng. 2019, 21, 5958904. [CrossRef]

40. Liu, Y.; Hao, Y.; Lu, Y.L. Improved Design of Risk Assessment Model for PPP Project under the Development of Marine Architecture. J. Coast. Res. 2018, 83, 74-80. [CrossRef]

41. Wibowo, A.; Kochendoerfer, B. Selecting BOT/PPP Infrastructure Projects for Government Guarantee Portfolio under Conditions of Budget and Risk in the Indonesian Context. J. Constr. Eng. Manag. 2011, 137, 512-522. [CrossRef]

42. Nguyen, D.A.; Garvin, M.J.; Gonzalez, E.E. Risk Allocation in US Public-Private Partnership Highway Project Contracts. J. Constr. Eng. Manag. 2018, 144, 04018017. [CrossRef]

43. Alireza, V.; Mohammadreza, Y.; Zin, R.M.; Yahaya, N. An enhanced multi-objective optimization approach for risk allocation in public-private partnership projects: A case study of Malaysia. Can. J. Civ. Eng. 2014, 41, 164-177. [CrossRef]

44. Chan, A.P.C.; Lam, P.T.I.; Wen, Y.; Ameyaw, E.E.; Wang, S. Cross-sectional analysis of critical risk factors for PPP water projects in China. J. Infrastruct. Syst. 2015, 21, 04014031. [CrossRef]

45. Ke, Y.; Wang, S.; Chan, A.P.C.; Cheung, E. Understanding the risks in China's PPP projects: Ranking of their probability and consequence. Eng. Constr. Archit. Manag. 2011, 18, 481-496. [CrossRef]

46. Effah Ameyaw, E.; Chan, A.P.C. Identifying public-private partnership (PPP) risks in managing water supply projects in Ghana. J. Facil. Manag. 2013, 11, 152-182. [CrossRef]

47. Ameyaw, E.E.; Chan, A.P. Risk ranking and analysis in PPP water supply infrastructure projects. Facilities 2015, 33, 428-453. [CrossRef]

48. $\mathrm{Xu}, \mathrm{Y}$. Critical risk factors affecting the implementation of PPP waste-to-energy projects in China. Appl. Energy 2015, 158, 403-411. [CrossRef]

49. Shrestha, A. Risks in PPP water projects in China: Perspective of local governments. J. Constr. Eng. Manag. 2017, 143, 05017006. [CrossRef]

50. Valipour, A.; Yahaya, N.; Md Noor, N.; Mardani, A.; Antuchevičienè, J. A new hybrid fuzzy cybernetic analytic network process model to identify shared risks in PPP projects. Int. J. Strateg. Prop. Manag. 2016, 20, 409-426. [CrossRef]

51. Wang, S.Q.; Dulaimi, M.F.Y.; Aguria, M. Risk management framework for construction projects in developing countries. Constr. Manag. Econ. 2004, 22, 237-252. [CrossRef]

52. Aladağ, H.; Işık, Z. Design and construction risks in BOT type mega transportation projects. Eng. Constr. Archit. Manag. 2019.

53. Valipour, A. A Fuzzy Analytic Network Process Method for Risk Prioritization In Freeway Ppp Projects: An Iranian Case Study. J. Civ. Eng. Manag. 2015, 21, 933-947. [CrossRef]

54. Jang, G.W. Bids-Evaluation Decision Model Development and Application for PPP Transport Projects: A Project Risks Modeling Framework; Colorado State University: Denver, CO, USA, 2010.

55. World Bank Group. Available online: https://ppi.worldbank.org/en/ppi (accessed on 29 August 2019).

56. China Public-Private Partnerships Center. Available online: http://www.cpppc.org/ (accessed on 29 August 2019).

57. Pinto, J.K.; Mantel, S.J. The causes of project failure. IEEE Trans. Eng. Manag. 1990, 37, 269-276. [CrossRef]

58. Jamali, D. Success and failure mechanisms of public private partnerships (PPPs) in developing countries: Insights from the Lebanese context. Int. J. Public Sector Manag. 2004, 17, 414-430. [CrossRef] 
59. Chan, A.P.C.; Chan, D.W.M.; Yeung, J.F.Y. Overview of the Application of "Fuzzy Techniques" in Construction Management Research. J. Constr. Eng. Manag. 2009, 135, 1241-1252. [CrossRef]

60. De, P.K.; Rawat, A. A Fuzzy Inventory Model Without Shortages Using Triangular Fuzzy Number. Fuzzy Inf. Eng. 2019, 3, 59-68. [CrossRef]

61. Zadeh, L.A. Fuzzy sets. Inf. Control 1965, 8, 338-353. [CrossRef]

62. Paek, J.H.; Lee, Y.W.; Ock, J.H. Pricing construction risk: Fuzzy set application. J. Constr. Eng. Manag. 1993, 119, 743-756. [CrossRef]

63. Zhang, G.; Zou, P.X.W. Fuzzy analytical hierarchy process risk assessment approach for joint venture construction projects in China. J. Constr. Eng. Manag. 2007, 133, 771-779. [CrossRef]

64. Abdelgawad, M.; Fayek, A.R. Fuzzy reliability analyzer: Quantitative assessment of risk events in the construction industry using fuzzy fault-tree analysis. J. Constr. Eng. Manag. 2011, 137, 294-302. [CrossRef]

65. Fahmi, A. Expected Values of Aggregation Operators on Cubic Triangular Fuzzy Number and Its Application to Multi-Criteria Decision Making Problems. Eng. Math. 2018, 2, 1-11. [CrossRef]

66. Wang, J. A synthetic method for knowledge management performance evaluation based on triangular fuzzy number and group support systems. Appl. Soft Comput. 2016, 39, 11-20. [CrossRef]

67. Chakraborty, D.; Jana, D.K.; Roy, T.K. A new approach to solve fully fuzzy transportation problem using triangular fuzzy number. Int. J. Oper. Res. 2016, 26, 153-179. [CrossRef]

68. Li, R.J. Fuzzy method in group decision making. Comput. Math. Appl. 1999, 38, 91-101. [CrossRef]

69. Wu, W.W.; Lee, Y.T. Developing global managers' competencies using the fuzzy DEMATEL method. Expert Syst. Appl. 2007, 32, 499-507. [CrossRef]

70. Govindan, K. Analysis of third party reverse logistics provider using interpretive structural modeling. Int. J. Prod. Econ. 2012, 140, 204-211. [CrossRef]

71. Mandal, A.; Deshmukh, S.G. Vendor Selection Using Interpretive Structural Modelling (ISM). Int. J. Oper. Prod. Manag. 1994, 14, 52-59. [CrossRef]

72. Govindan, K.; Shankar, K.M.; Kannan, D. Application of fuzzy analytic network process for barrier evaluation in automotive parts remanufacturing towards cleaner production-A study in an Indian scenario. J. Clean. Prod. 2016, 114, 199-213. [CrossRef]

73. Luthra, S. Adoption of smart grid technologies: An analysis of interactions among barriers. Renew. Sustain. Energy Rev. 2014, 33, 554-565. [CrossRef]

74. Tseng, C.P.; Chen, C.W.; Tu, Y.P. A new viewpoint on risk control decision models for natural disasters. Nat. Hazards 2011, 59, 1715-1733. [CrossRef]

75. Yanmei, L.; Yanmei, L.; Zi, C. Analysis of load factors based on interpretive structural model. J. Comput. 2012, 7, 1704-1711.

76. Li, Y. Risks assessment in thermal power plants using ISM methodology. Ann. Oper. Res. 2019, 279, 89-113. [CrossRef]

77. Yadav, D.K.; Barve, A. Analysis of critical success factors of humanitarian supply chain: An application of Interpretive Structural Modeling. Int. J. Disaster Risk Reduct. 2015, 12, 213-225. [CrossRef]

78. Chakraborty, K.; Mondal, S.; Mukherjee, K. Critical analysis of enablers and barriers in extension of useful life of automotive products through remanufacturing. J. Clean. Prod. 2019, 227, 1117-1135. [CrossRef]

79. Tseng, M.L.; Lim, M.K.; Wu, K.J. Improving the benefits and costs on sustainable supply chain finance under uncertainty. Int. J. Prod. Econ. 2019, 218, 308-321. [CrossRef]

80. Chaudhuri, A. Risk propagation and its impact on performance in food processing supply chain: A fuzzy interpretive structural modeling based approach. J. Model. Manag. 2016, 11, 660-693. [CrossRef]

81. Ragade, R.K. Fuzzy interpretive structural modeling. J. Cybern. 1976, 6, 189-211. [CrossRef]

82. Bhosale, V.A.; Kant, R. An integrated ISM fuzzy MICMAC approach for modelling the supply chain knowledge flow enablers. Int. J. Prod. Res. 2016, 54, 7374-7399. [CrossRef]

83. Han, Q. Public private partnership in brownfield remediation projects in China: Identification and structure analysis of risks. Land Use Policy 2019, 84, 87-104. [CrossRef]

84. Zhang, L. Risk identification and analysis for PPP projects of electric vehicle charging infrastructure based on 2-tuple and the DEMATEL model. World Electr. Veh. J. 2019, 10, 4. [CrossRef]

85. Wu, Y. Risk management of public-private partnership charging infrastructure projects in China based on a three-dimension framework. Energy 2018, 165, 1089-1101. [CrossRef] 
86. Li, Y.; Wang, X.Y. Risk assessment for public-private partnership projects: Using a fuzzy analytic hierarchical process method and expert opinion in China. J. Risk Res. 2018, 21, 952-973. [CrossRef]

87. Ke, Y.J.; Wang, S.Q.; Chan, A.P.C. Risk Misallocation in Public-Private Partnership Projects in China. Int. Public Manag. J. 2013, 16, 438-460. [CrossRef]

88. Wang, W. Assessing contributory factors in potential systemic accidents using AcciMap and integrated fuzzy ISM-MICMAC approach. Int. J. Ind. Ergon. 2018, 68, 311-326. [CrossRef]

89. Chan, A.P.C. Empirical Study of Risk Assessment and Allocation of Public-Private Partnership Projects in China. J. Manag. Eng. 2011, 27, 136-148. [CrossRef]

90. The National People's Congress of the People's Republic of China. Environmental protection tax law of the People's Republic of China. 2018. Available online: http://www.npc.gov.cn/npc/c12435/201811/ $5 \mathrm{e} 7 \mathrm{~d} 3 \mathrm{cfb} 3 \mathrm{afa} 4 \mathrm{ef79428c0ff7a29fdd7.shtml} \mathrm{(accessed} \mathrm{on} 29$ August 2019).

91. Zhang, S. PPP application in infrastructure development in China: Institutional analysis and implications. Int. J. Proj. Manag. 2015, 33, 497-509. [CrossRef]

92. Chen, C.; Li, D.; Man, C.X. Toward Sustainable Development? A Bibliometric Analysis of PPP-Related Policies in China between 1980 and 2017. Sustainability 2019, 11, 142. [CrossRef]

93. Wojewnik-Filipkowska, A.; Wegrzyn, J. Understanding of Public-Private Partnership Stakeholders as a Condition of Sustainable Development. Sustainability 2019, 11, 1194. [CrossRef]

94. Li, L. Enhanced Cooperation among Stakeholders in PPP Mega-Infrastructure Projects: A China Study. Sustainability 2018, 10, 2791. [CrossRef]

95. Henjewele, C.; Fewings, P.; Rwelamila, P.D. De-marginalising the public in PPP projects through multi-stakeholders management. J. Financ. Manag. Prop. Constr. 2013, 18, 210-231. [CrossRef]

(C) 2019 by the authors. Licensee MDPI, Basel, Switzerland. This article is an open access article distributed under the terms and conditions of the Creative Commons Attribution (CC BY) license (http://creativecommons.org/licenses/by/4.0/). 